\title{
Wolbachia and the insect immune system: what reactive oxygen species can tell us about the mechanisms of Wolbachia-host interactions
}

\author{
Roman Zug* and Peter Hammerstein \\ Institute for Theoretical Biology, Humboldt-Universität zu Berlin, Berlin, Germany
}

Wolbachia are intracellular bacteria that infect a vast range of arthropod species, making them one of the most prevalent endosymbionts in the world. Wolbachia's stunning evolutionary success is mostly due to their reproductive parasitism but also to mutualistic effects such as increased host fecundity or protection against pathogens. However, the mechanisms underlying Wolbachia phenotypes, both parasitic and mutualistic, are only

OPEN ACCESS

Edited by:

Puttaraju H. P.

Bangalore University, India

Reviewed by:

Steven G. Ball,

Université des Sciences

et Technologies de Lille, France

Fabrice Vavre,

Université Claude Bernard Lyon 1,

France

*Correspondence:

Roman Zug

roman.zug@biologie.hu-berlin.de

Specialty section:

This article was submitted to

Evolutionary and Genomic

Microbiology,

a section of the journal

Frontiers in Microbiology

Received: 30 June 2015

Accepted: 15 October 2015

Published: 27 October 2015

Citation:

Zug R and Hammerstein P (2015)

Wolbachia and the insect immune

system: what reactive oxygen species

can tell us about the mechanisms

of Wolbachia-host interactions.

Front. Microbiol. 6:1201.

doi: 10.3389/fmicb.2015.01201 poorly understood. Moreover, it is unclear how the insect immune system is involved in these phenotypes and why it is not more successful in eliminating the bacteria. Here we argue that reactive oxygen species (ROS) are likely to be key in elucidating these issues. ROS are essential players in the insect immune system, and Wolbachia infection can affect ROS levels in the host. Based on recent findings, we elaborate a hypothesis that considers the different effects of Wolbachia on the oxidative environment in novel vs. native hosts. We propose that newly introduced Wolbachia trigger an immune response and cause oxidative stress, whereas in coevolved symbioses, infection is not associated with oxidative stress, but rather with restored redox homeostasis. Redox homeostasis can be restored in different ways, depending on whether Wolbachia or the host is in charge. This hypothesis offers a mechanistic explanation for several of the observed Wolbachia phenotypes.

Keywords: Wolbachia, reactive oxygen species, immune system, insects, symbiont-host interactions

\section{INTRODUCTION}

Reactive oxygen species (ROS) have long been viewed as purely harmful molecules contributing to oxidative stress, which can cause severe cell damage. On the other hand, ROS can also play a beneficial role, for example in intracellular signaling and innate immune defense. Intracellular bacteria of the genus Wolbachia have evolved intriguing capabilities to manipulate the biology of their arthropod hosts. Wolbachia have recently been shown to influence ROS production and the oxidative environment as a whole, suggesting an involvement of ROS in Wolbachia-induced phenotypes. In this article, we briefly review the relevant facts about ROS and give an overview of the insect immune response with a focus on ROS. In the main part, we outline the interactions between Wolbachia and the host immune system. We explore the possible roles of ROS in different Wolbachia phenotypes and hypothesize how interference with the host oxidative environment has shaped various aspects of the Wolbachia-insect symbiosis. Finally, we discuss some corollaries of the hypothesis. 


\section{REACTIVE OXYGEN SPECIES, OXIDATIVE STRESS, AND REDOX HOMEOSTASIS}

After the advent of molecular oxygen in the Earth's atmosphere, organisms evolved metabolic mechanisms that use oxygen to produce energy. In eukaryotic cells, aerobic respiration takes place in the mitochondria. During oxidative phosphorylation, electrons are transferred along the mitochondrial respiratory chain to generate a proton gradient which eventually enables the synthesis of ATP. In this electron transport chain, the final acceptor of electrons is molecular oxygen which thereby is reduced to produce water. Occasionally, however, oxygen is prematurely and incompletely reduced, giving rise to superoxide. The superoxide anion belongs to a class of oxygen-derived molecules that readily oxidize other molecules and are commonly referred to as ROS. In addition to superoxide, ROS include hydrogen peroxide, hypochlorous acid, hydroxyl radical, singlet oxygen, and ozon. It has been known for a long time that an excessive load of ROS damages diverse cellular macromolecules, including proteins, lipids, and DNA, a process known as oxidative stress. The concept of oxidative stress has its roots in the mid-twentieth century when researchers began to explore the harmful effects of oxidizing free radicals (Gerschman et al., 1954) and their possible involvement in the aging process (Harman, 1956). A couple of years later, the antioxidant enzyme superoxide dismutase (SOD) was discovered which eliminates superoxide from the cell and thus protects the cell from its toxicity (McCord and Fridovich, 1969; McCord et al., 1971). Antioxidant enzymes aimed at detoxifying hydrogen peroxide include glutathione peroxidase $(\mathrm{GPx})$, thioredoxin peroxidase (TPx), which is a member of the Peroxiredoxin (Prx) family, and catalase. Since the discovery of antioxidants, oxidative stress has been invoked as contributing to a number of diseases, including cancer (Storz, 2005; Valko et al., 2006), cardiovascular diseases (Griendling and FitzGerald, 2003), neurodegenerative disorders (Barnham et al., 2004), and diabetes (Maritim et al., 2003). Oxidative stress also negatively affects male and female reproductive ability, and detoxification of ROS by antioxidants is required for maintaining fertility/fecundity in Drosophila (Parkes et al., 1998), mosquitoes (DeJong et al., 2007), and mammals (Ho et al., 1998). In general, therefore, ROS have long been seen as harmful but unavoidable by-products of an aerobic lifestyle.

It therefore came as a surprise when enzymes were discovered whose sole function is the production of ROS (Royer-Pokora et al., 1986; Suh et al., 1999). The membrane-bound enzymes NADPH oxidases (NOX1-5) and dual oxidases (DUOX1-2) catalyze the reduction of molecular oxygen to generate superoxide and/or hydrogen peroxide, using NADPH as an electron donor (Brown and Griendling, 2009). DUOX enzymes can be distinguished from NOX enzymes by the presence of an extracellular peroxidase homology domain (PHD), in addition to the intracellular NADPH oxidase domain (Lambeth, 2004). Commonly, however, the term NOX is used for the whole seven-member protein family. NOX enzymes are expressed in a diverse array of cells and tissues and are present in most eukaryotes (Bedard et al., 2007; Aguirre and Lambeth, 2010). Therefore, the view that ROS are purely harmful by-products of mitochondrial metabolism needed reconsideration. It is important to note, though, that despite the existence of ROS-producing enzymes, the vast majority of cellular ROS (estimated at approximately 90\%) can be traced back to a mitochondrial origin (Balaban et al., 2005). Nevertheless, the fact that ROS are actively synthesized prompted research into their possible biological functions. It is now clear that ROS, both those produced within mitochondria and those generated by NOX enzymes, act as important signaling molecules in diverse physiological processes. As such, ROS are involved in regulating cellular homeostasis, stem cell proliferation and differentiation, cell motility and migration, autophagy, cell death and aging, and, last but not least, immunity and host defense (D’Autréaux and Toledano, 2007; Hamanaka and Chandel, 2010; Finkel, 2011; Ray et al., 2012; Sena and Chandel, 2012; Nathan and Cunningham-Bussel, 2013; Holmström and Finkel, 2014; Lambeth and Neish, 2014; Schieber and Chandel, 2014; Reczek and Chandel, 2015). Therefore, organisms must tightly control the balance between ROS production and degradation. This finetuned balance between oxidants and antioxidants is called redox homeostasis.

\section{INSECT IMMUNITY: ANTIMICROBIAL PEPTIDES, ROS, AND AUTOPHAGY}

The innate immune response of insects consists of multiple defense mechanisms, including epithelial barriers and both local and systemic immune reactions. Most research in insect immunity has focused on Drosophila melanogaster (Lemaitre and Hoffmann, 2007; Buchon et al., 2014; but see Rolff and Reynolds, 2009, for a broader perspective). The cellular immune response is executed by hemocytes and emcompasses several distinct mechanisms, including phagocytosis, encapsulation, coagulation, and melanization (Jiravanichpaisal et al., 2006; Lemaitre and Hoffmann, 2007; Strand, 2008; Fauvarque and Williams, 2011). Some of these mechanisms (encapsulation, melanization) involve the generation of ROS at infection sites to kill pathogens (Nappi et al., 1995; Nappi and Vass, 1998; Kumar et al., 2003). At the core of the systemic immune response lies the production of antimicrobial peptides (AMPs) by the fat body and their subsequent release into the hemolymph (for an overview of insect AMPs, see Yi et al., 2014). AMP gene expression is mainly controlled by two distinct signaling pathways, the Toll pathway and the Imd pathway, both of which include homologs of the NF- $\kappa$ B pathway (Khush et al., 2001; Brennan and Anderson, 2004; Ferrandon et al., 2007; Lemaitre and Hoffmann, 2007; Hetru and Hoffmann, 2009). The Imd pathway is predominantly activated by Gram-negative bacteria, whereas Gram-positive bacteria, fungi, and yeast trigger the Toll pathway (Buchon et al., 2014). In the lab, systemic responses have frequently been elicited by bacterial injection into the hemocoel. However, this might not reflect the natural way of infection. Commonly, epithelia such as those lining the gut are the first barrier a pathogen encounters when infecting the host. A peculiarity of gut epithelia is the fact that they not only are in constant contact with pathogens, but also host a number of beneficial commensal bacteria, the so-called gut microbiota. Commensal gut microbes are involved in diverse physiological functions of 
their hosts, including organ development and morphogenesis, host metabolism, and immunity (Sommer and Bäckhed, 2013; for reviews on the Drosophila/insect gut microbiota, see, for example, Buchon et al., 2013; Engel and Moran, 2013; Erkosar et al., 2013; Lee and Brey, 2013). The challenge for the host immune system, therefore, is to find the balance between fighting pathogens and tolerating the microbiota (Sansonetti and Medzhitov, 2009).

Accordingly, a tight regulation of the production of immune effector molecules is strictly needed. In the Drosophila gut, there are two major classes of immune effectors, AMPs and ROS (Ryu et al., 2010; Kuraishi et al., 2013). AMP generation in the gut is controlled by the Imd pathway, but not by the Toll pathway (Tzou et al., 2000). The Imd pathway is triggered when the bacterial cell wall component diaminopimelic acid (DAP)-type peptidoglycan (PG) is recognized by $P G$ recognition proteins (PGRPs) in the host membrane (Leulier et al., 2003; Bosco-Drayon et al., 2012; Neyen et al., 2012; for reviews on PGRPs, see Royet and Dziarski, 2007; Royet et al., 2011). In the absence of pathogenic bacteria, PGtriggered AMP gene expression is repressed by negative regulators of the Imd pathway to protect the commensal microbiota, thereby maintaining the balance between immune tolerance and immune response (Lhocine et al., 2008; Ryu et al., 2008; Paredes et al., 2011; Bosco-Drayon et al., 2012; Bonnay et al., 2013; Dantoft et al., 2013).

Local production of AMPs only seems to constitute a complementary response against microbes that are resistant against ROS (Ryu et al., 2006), the second major immune effector class in the Drosophila gut. Indeed, DUOX-dependent production of microbicidal ROS serves as the first line of defense in gut immunity (Ha et al., 2005a, 2009a). It is assumed that the NADPH oxidase domain of DUOX synthesizes $\mathrm{H}_{2} \mathrm{O}_{2}$, which the PHD then converts into the highly microbicidal $\mathrm{HOCl}$ in the presence of chloride (Ha et al., 2005a). Infection-induced ROS generation in the Drosophila gut can also act as a signal for AMP production in the fat body, thus triggering a systemic immune response (Wu et al., 2012). After the pathogen-induced increase in ROS production, ROS levels are actively reduced by immune-regulated catalase (IRC) activity to avoid excessive oxidative stress (Ha et al., 2005b).

DUOX-dependent ROS production in the Drosophila gut is regulated by two signaling pathways (Bae et al., 2010): The enzymatic activity of DUOX is controlled by the G $\alpha$ q-PLC $\beta$ $\mathrm{Ca}^{2+}$ pathway ("DUOX activity pathway"; Ha et al., 2009a), while DUOX gene expression is regulated by a MEKK1-MKK3-p38ATF2 pathway ("DUOX expression pathway"; Ha et al., 2009b, Chakrabarti et al., 2014). Activation of both pathways is required for stable ROS production. Interestingly, $\mathrm{PG}$ is able to activate the DUOX expression pathway, but not the DUOX activity pathway. Therefore, DUOX-dependent ROS generation cannot depend on PG alone (Ha et al., 2009a,b; Bae et al., 2010). Recently, bacterialderived uracil was identified as a non-PG ligand triggering DUOX-dependent ROS generation (Lee et al., 2013). Uracil is probably recognized by a G-protein-coupled receptor (GPCR) and, via Hedgehog-induced signaling endosomes, induces PLC $\beta$ dependent $\mathrm{Ca}^{2+}$ mobilization which triggers DUOX activation (Lee et al., 2015). Strikingly, uracil is released by pathogenic bacteria, but not by commensal symbionts (Lee et al., 2013). This allows the gut epithelia to distinguish between pathogens and commensal bacteria, thus maintaining immune homeostasis in the Drosophila gut (Kim and Lee, 2014; You et al., 2014).

What do we know about the involvement of ROS in innate immunity and host defense in insects other than Drosophila? The production of ROS as a countermeasure to bacterial and/or fungal infection has been reported from species as diverse as the cockroach Blaberus discoidalis (Blattodea; Whitten and Ratcliffe, 1999), the silkworm Bombyx mori (Lepidoptera; Ishii et al., 2008), the scale insect Dactylopius coccus (Hemiptera; García-Gil de Muñoz et al., 2007), the greater wax moth Galleria mellonella (Lepidoptera; Bergin et al., 2005), the sand fly Lutzomyia longipalpis (Diptera; Diaz-Albiter et al., 2012), the tiger moth Parasemia plantaginis (Lepidoptera; Mikonranta et al., 2014), and the cattle tick Rhipicephalus microplus (Ixodida; Pereira et al., 2001). Hematophagous insects may also become infected by blood-borne parasites, e.g., the malaria parasite Plasmodium. The mosquito Anopheles gambiae is one of the most efficient malaria vectors known. Interestingly, sufficiently high ROS levels are required for An. gambiae to mount an effective immune response against Plasmodium and bacteria (Kumar et al., 2003; MolinaCruz et al., 2008). Elevated ROS levels to fight off Plasmodium can be generated by mitochondria in mosquito midgut cells (Gonçalves et al., 2012) or by an Enterobacter bacterium from the An. gambiae gut microbiota (Cirimotich et al., 2011). Therefore, host defense against bacterial, fungal, and Plasmodium infection based on ROS is widespread among various insect species.

Insect immunity based on the production of AMPs and ROS (controlled by the Toll pathway, the Imd pathway, and both DUOX pathways) is able to fight off both Gram-positive and Gram-negative bacteria, fungi, yeast, and protozoa such as Plasmodium (Carter and Hurd, 2010; Buchon et al., 2014). AMP production based on the Toll/Imd pathways may also be involved in the antiviral response, in addition to RNA interference and other mechanisms (Xi et al., 2008b; Sabin et al., 2010; Merkling and van Rij, 2013; Ferreira et al., 2014; Lamiable and Imler, 2014). To the best of our knowledge, however, nothing is known about ROS as antiviral effectors in a natural insect system (but see Wang et al., 2001, and below). In general, insect host defenses against intracellular pathogens (such as viruses) are less well studied than those against extracellular pathogens. AMPs have been shown to control obligate intracellular bacteria such as Rickettsia and Anaplasma (Baldridge et al., 2005; Liu et al., 2012b). Moreover, several immune responses are known that specifically target intracellular pathogens (Steinert and Levashina, 2011; Lundgren and Jurat-Fuentes, 2012; Péan and Dionne, 2014). Autophagy seems to represent a general and evolutionarily conserved defense mechanism against intracellular pathogens (Virgin and Levine, 2009; Deretic, 2010; Nakamoto et al., 2012; Yuk et al., 2012; Choy and Roy, 2013; Deretic et al., 2013). In Drosophila, for example, one type of PGRP (PGRP-LE) acts as an intracellular receptor for DAP-type PG and thus as an intracellular sensor of Gramnegative bacteria (Kaneko et al., 2006). PGRP-LE also induces an autophagic response to prevent the intracellular growth of bacterial pathogens, and this induction occurs independently of the Toll and Imd pathways (Yano et al., 2008; Kurata, 2010). Moreover, autophagy is also activated and regulated by ROS 
(Huang et al., 2009; Scherz-Shouval and Elazar, 2011; Sena and Chandel, 2012). In sum, several distinct and yet interconnected immune responses are at work to defend the insect host against a plethora of different pathogens.

\section{WOLBACHIA AND THE INSECT IMMUNE SYSTEM}

Wolbachia are maternally transmitted intracellular Gram-negative bacteria that infect a vast range of arthropod species, probably making them the most prevalent endosymbionts in the world (Hilgenboecker et al., 2008; Zug and Hammerstein, 2012; Weinert et al., 2015). Wolbachia's evolutionary success is generally attributed to their reproductive parasitism, which ensures their vertical transmission from mother to offspring (Stouthamer et al., 1999; Werren et al., 2008). These reproductive manipulations include male killing, feminization, parthenogenesis induction, and cytoplasmic incompatibility (CI). The latter phenotype seems to be the most frequent one and occurs if males infected with CI-Wolbachia mate with uninfected females; these matings suffer from high offspring mortality. Infected females, in contrast, can mate successfully with both uninfected and infected males. In sum, all reproductive manipulations enhance the proportion of infected females and thus benefit the maternally inherited Wolbachia. Recently, however, two additional facets of the biology of Wolbachia are increasingly acknowledged to contribute to their success: horizontal transmission and mutualistic effects. Transmission between different host species is likely to be a major reason for Wolbachia's vast abundance, particularly if it occurs over large phylogenetic distances (Zug et al., 2012). In addition, the global spread of Wolbachia might be facilitated by mutualistic effects such as increased host fecundity and longevity or protection against pathogens (Zug and Hammerstein, 2015). Nevertheless, reproductive parasitism seems to be more prevalent than mutualism in arthropod hosts, and it is frequently associated with a fitness cost to the host (Zug and Hammerstein, 2015).

What does the host defense against Wolbachia infection look like? In principle, hosts can employ two different strategies to defend themselves against infections: resistance and tolerance. Resistance is the ability to clear the infection, while tolerance is the ability to reduce the fitness costs of infection, without clearing the infection itself (Schneider and Ayres, 2008). Whether a host responds to Wolbachia through resistance or tolerance strongly depends on two features of the infection: its age and its phenotypic effects. A recently acquired infection is likely to trigger an immune response, which is the key resistance mechanism. In coevolved associations, by contrast, resistance may no longer be the best response to infection. Whether or not resistance is the host's best option in coevolved symbioses mainly depends on the symbiont's phenotype. Reproductive manipulations such as feminization and male killing reduce host fitness and thus are expected to lead to the evolution of resistance. Indeed, host suppressor alleles have been identified that confer resistance to feminizing and male killing Wolbachia (Rigaud and Juchault, 1992; Hornett et al., 2006). With other Wolbachia phenotypes, things are a bit more complex. In the case of CI, infected females are "addicted" to Wolbachia-if they lose the symbionts, their offspring will suffer from high mortality rates when fathered by infected males. Therefore, females infected with CI-Wolbachia are selected to maintain the bacteria and even increase the efficiency of maternal transmission. On the other hand, suppressor genes are predicted to spread in males, and successive selection for male suppressors of Wolbachia should lead to long-term elimination of infection (Koehncke et al., 2009). With respect to Wolbachia-induced parthenogenesis, the symbiont has gone to fixation in most populations that are infected. In these populations there are no males, and females depend on the bacteria for asexual reproduction. Under such circumstances of host dependence, infected females are not expected to evolve mechanisms of resistance ("dependence" barrier to resistance; Zug and Hammerstein, 2015). However, nuclear suppressor alleles have been hypothesized for populations where infected and uninfected individuals coexist (Huigens, 2003). Finally, if Wolbachia exhibits a mutualistic phenotype, evolution of resistance will also be selected against ("fitness benefit" barrier to resistance; Zug and Hammerstein, 2015). When resistance is not feasible, tolerance mechanisms represent an alternative host strategy to deal with the infection. The evolution of tolerance is associated with the attenuation of the immune response that originally was there to eliminate the bacteria. Immune tolerance is also an efficient means to reduce the risk that host tissue is damaged as a side effect of the immune response (immunopathology).

In summary, the evolution of host resistance is expected in many, but not all, Wolbachia-host associations. In those associations in which resistance evolution is expected, Wolbachia should, in principle, trigger the host immune system which should aim at eliminating the bacteria, regardless of whether they are novel or native. On the other hand, given the huge number of infected insect species and the recurrent occurrence of successful transmission into novel host species, why is the host defense machinery not more efficient in overcoming the infection? Have Wolbachia evolved mechanisms to suppress or interfere with the immune system, or do they hide from it? Or does the high prevalence of Wolbachia indicate that, frequently, hosts are not selected to evolve resistance (but rather tolerance)? In the following paragraphs, we outline in more detail the interplay between Wolbachia infection and the different host defense mechanisms, with special emphasis on the host oxidative environment.

\section{Wolbachia and AMP-/Autophagy-Based Immunity}

Interestingly, in their native hosts, Wolbachia do not induce AMP gene expression, as has been shown for Aedes albopictus, D. melanogaster, Drosophila simulans, and Tetranychus urticae (Bourtzis et al., 2000; Wong et al., 2011; Rancès et al., 2012; Zhang et al., 2015). On the other hand, Wolbachia-infected D. simulans and Ae. albopictus are still able to activate AMP gene expression when challenged by other bacterial pathogens, e.g., E. coli (Bourtzis et al., 2000). These results suggest that Wolbachia neither induce nor suppress the AMP-based branch of the immune system of their natural hosts. Drosophila species seem to be naturally infected with only two maternally inherited 




bacteria, Wolbachia and Spiroplasma (Mateos et al., 2006). In Spiroplasma-infected $D$. melanogaster, the same picture emerges: in their natural host, the bacteria neither upregulate nor downregulate the expression of AMP genes (Hurst et al., 2003; Hutchence et al., 2011). Taken together, these findings suggest that endosymbionts such as Wolbachia have evolved means to evade the host immune system by stealth (Figure 1D; Siozios et al., 2008). This notion is corroborated by the fact that, in the host cytoplasm, Wolbachia are located within vesicles whose outermost membrane is of host origin (Louis and Nigro, 1989). This probably helps the bacteria to hide from the host immune system. Another possible reason for the lack of Wolbachiainduced AMP upregulation is that the host has shut down the AMP-based immune response when selection favors the maintenance of the bacteria (Figure 1G). However, it is unclear how this immune tolerance could be restricted to Wolbachia so that other pathogens are still effectively targeted. This problem could be resolved by the fact that AMPs do not need to be shut down for ensuring immune tolerance in coevolved symbioses, but instead are actively involved in symbiont maintenance (Login et al., 2011).

The fact that Wolbachia do not elicit an AMP-based immune response in their native hosts stands in stark contrast to the strong induction of AMP gene expression when Wolbachia are introduced into novel hosts (Figure 1A; Xi et al., 2008a; Kambris et al., 2009, 2010; Moreira et al., 2009; Bian et al., 2010). This is indicative of a systemic immune response triggered by the canonical Toll and/or Imd pathway (immune upregulation; note that the term immune priming is equivalent to such general immune upregulation only in its unspecific meaning; compare, for example, Roth et al., 2009 and Masri and Cremer, 2014 for a different usage of the term). As Gram-negative bacteria, newly introduced Wolbachia are probably detected by the Imd pathway that is triggered by recognition of DAP-type PG from the bacterial cell wall. Although Wolbachia lack a proper cell wall and PG has never been detected, they are probably able to synthesize DAP (Dunning Hotopp et al., 2006; Vollmer et al., 2013). Moreover, it was recently shown that the PG-associated lipoprotein (PAL) 
is located on the cell membrane of Wolbachia (Voronin et al., 2014). PAL is known to specifically bind DAP (Parsons et al., 2006). Therefore, DAP is present on the Wolbachia membrane, and perhaps this is sufficient to be recognized by PGRPs which then trigger the Imd pathway and subsequent AMP generation.

The discovery of PGRP-LE as an intracellular sensor of DAP-type PG (Kaneko et al., 2006) also opens the possibility of an autophagic immune defense against Wolbachia. It was recently shown that Wolbachia induce the autophagy pathway in a naturally infected Ae. albopictus cell line (Voronin et al., 2012). Hence, one might expect bacterial strategies to counteract autophagy. Indeed, autophagy-associated genes are downregulated in the ovaries of two hosts naturally infected with Wolbachia, the woodlouse Armadillidium vulgare and the wasp Asobara tabida, supporting the notion that the symbionts suppress the autophagic signal to prevent their elimination (Figure 1E; Chevalier et al., 2012; Kremer et al., 2012). Again, it is also conceivable that the host itself is responsible for the downregulation-another possible case of evolved immune tolerance when symbiont presence is favored (Figure 1H). In contrast to these coevolved associations, a transfected Wolbachia strain causes a catastrophic autophagic response in another woodlouse, Porcellio d. dilatatus, resulting in the death of the new host (Figure 1B; Le Clec'h et al., 2012). Therefore, an autophagic immune response is observable in novel, but not in native hosts, mirroring the situation with regard to AMP-based immune defense.

How can we reconcile these differing findings concerning the immune response to Wolbachia in native vs. novel hosts? Perhaps, it is not too surprising that Wolbachia do elicit an immune response in novel hosts. In insects that acquired Wolbachia only recently (either by natural or artificial means), the bacteria are recognized as foreign, probably by PGRPs detecting DAP on Wolbachia membranes, and then AMP- and/or autophagyassociated defense mechanisms are triggered to eliminate the infection (Figures 1A,B). With ongoing coevolution, however, Wolbachia have found ways to prevent their elimination, for example by evading the AMP-based immune response (immune evasion by stealth; Figure 1D) and by suppressing the autophagyassociated immune defense (immune suppression; Figure 1E). Alternatively, evolution of immune tolerance enables the host to reduce costly defense mechanisms when selection favors the presence of Wolbachia (Figures 1G,H).

\section{Wolbachia and ROS-Based Immunity}

Given their vertical transmission through the female germline and their reproductive manipulations, Wolbachia are expected to reside primarily in the host reproductive tissues. Although this is true, they are also able to infect somatic tissues, including tissues of immunological importance, such as the gut, fat body, and hemolymph (Dobson et al., 1999; Cheng et al., 2000; Ijichi et al., 2002; Zouache et al., 2009; Frost et al., 2014). In gut epithelia, AMPs represent only one of two major classes of immune effectors, the other one being ROS (see above). Therefore, when asking about the relationship between Wolbachia infection and the host immune system, it is also important to consider possible interactions between Wolbachia on the one hand and ROS and the oxidative environment on the other hand, in particular if infection in the gut has been reported.

The first, indirect evidence of such an interaction between Wolbachia and the host oxidative environment came from studies on the role of mitochondria in various aspects of host biology. In D. melanogaster, tetracycline treatment to eliminate Wolbachia resulted in a significant decrease in lipid hydroperoxide, a marker for ROS-induced oxidative damage (Driver et al., 2004). However, this finding could be due to a direct negative effect of tetracycline on mitochondrial efficiency (Ballard and Melvin, 2007). Further indirect evidence comes from the fact that Wolbachia infection can have a profound influence on mitochondrial DNA (mtDNA) haplotype diversity (Hurst and Jiggins, 2005), and different mtDNA haplotypes can differ in mitochondrial ROS production rates (Ballard, 2005).

Brennan et al. (2008) were the first to demonstrate a more direct effect of Wolbachia on the host oxidative environment. The mosquito Ae. albopictus is naturally infected with CI-inducing Wolbachia. In an Ae. albopictus cell line, the authors found that Wolbachia infection is associated with high levels of ROS (as compared to an identical cell line treated with the antibiotic rifampicin). These ROS probably are a product of the host immune response (although they may also be a side-product of bacterial metabolism). In addition, Wolbachia infection is associated with the upregulation of several host antioxidant genes. These antioxidant proteins include copper-zinc SOD1, Prx5, and GPx. Antioxidant upregulation may be a host countermeasure to mitigate the negative effects of increased ROS levels. However, as the authors point out, there is so far only little support in the literature for antioxidant upregulation as a host response to pathogen infection. Alternatively, one might speculate that Wolbachia induce the host antioxidant system in order to be protected against the host immune response based on increased ROS levels. Possibly, induction of the host antioxidant system is due to effectors secreted by the bacterial type IV secretion system (T4SS). The Wolbachia T4SS is a potential pathway to transfer effector proteins into the host cytoplasm and therefore might be involved in Wolbachia-induced host phenotypes (Pichon et al., 2009). Recently, a T4SS effector in Ehrlichia (a close relative of Wolbachia) was shown to be translocated to mitochondria and to upregulate a host SOD (MnSOD), thereby reducing ROS levels and apoptosis (Liu et al., 2012a). Lastly, Wolbachia also seem to be able to produce their own antioxidants to protect themselves, since two bacterial antioxidant proteins were identified as well, a bacterial type of SOD (Fe-SOD) and bacterioferritin (Bfr). Iron $(\mathrm{Fe})$ is an essential element for most organisms, but also a cause of oxidative stress as it catalyzes the generation of highly reactive hydroxyl radicals (Fenton reaction; Nappi and Vass, 2002). Hence, bacterioferritin has important functions both in bacterial iron storage and, although not commonly referred to as an antioxidant, in fighting iron-mediated oxidative stress (Carrondo, 2003). Upregulation of Wolbachia bacterioferritin expression under ironinduced stress was also observed in naturally infected $D$. simulans (Kremer et al., 2009). Given the crucial role of iron at the interface of immunity, infection and host-pathogen interactions (Cassat and Skaar, 2013; Nairz et al., 2014), Wolbachia's ability 
to interfere with host iron metabolism might be an important factor underlying diverse phenotypes and thus contributing to the unparalleled success of Wolbachia (Gill et al., 2014).

\section{Wolbachia and the Oxidative Environment: A Hypothesis}

Based on the results by Brennan et al. (2008), on subsequent propositions regarding the possible involvement of Wolbachia in the host oxidative environment (Kremer et al., 2010; Moné et al., 2014), and on the findings concerning AMP-/autophagybased immunity, we propose the following hypothesis. In novel hosts, Wolbachia induce a ROS-based immune response, leading to oxidative stress (Figure 1C). In general, therefore, we expect infections in novel hosts to be associated with a disruption of redox homeostasis (although Wolbachia effects on antioxidant production are hardly predictable). In native hosts, by contrast, Wolbachia infection is expected to be associated with restored redox homeostasis, resulting from coevolutionary processes between symbiont and host. Redox homeostasis can be restored by Wolbachia or by the host (or by a combination of both) because both benefit from reduced oxidative stress. In the first case, Wolbachia not only induce a ROS-based immune response, but also the expression of antioxidant genes (regardless of whether these genes are part of the symbiont or host genome). In doing so, the bacteria interfere with the host immune response (immune interference) and are involved in maintaining redox homeostasis (Figure 1F). This may be particularly relevant if there are additional sources of oxidative stress (e.g., iron overload). In the second case, the host decreases the Wolbachia-induced immune response by reducing ROS production or by increasing antioxidant production (immune tolerance), and thereby restores redox homeostasis itself (Figure 1I). In what follows, we will gather further evidence in support of this hypothesis.

\section{Wolbachia and the Oxidative Environment in Novel Hosts}

Several studies report effects of Wolbachia infection on the oxidative environment of arthropods that are naturally either uninfected of infected with a different strain. Examples where a novel Wolbachia infection causes an increase in ROS levels include the mosquitoes Aedes aegypti (Pan et al., 2012), Aedes polynesiensis (Andrews et al., 2012), and Anopheles stephensi (Bian et al., 2013). All mosquitoes were transfected with the Wolbachia strain $w \mathrm{AlbB}$, which naturally infects Ae. albopictus. In Ae. aegypti, ROS production was shown to be due to the upregulation of NADPH oxidase (NOXM) and dual oxidase (DUOX2), the latter one being upregulated 28 -fold (Pan et al., 2012). Interestingly, the authors found that increased ROS levels activate the Toll pathway, leading to the production of AMPs and antioxidants. The fact that Wolbachia induce both the activation of ROS and antioxidants in Ae. aegypti is reminiscent of the situation in evolved symbioses (immune interference by Wolbachia). Given the relatively close phylogenetic relationship between donor (Ae. albopictus) and recipient (Ae. aegypti), it might not be too difficult for $w$ AlbB to induce antioxidant production in Ae. aegypti and thus establish redox homeostasis in a novel host. On the other hand, some studies involving transfected cell lines show the downregulation of antioxidants as a result of infection (Xi et al., 2008a; Hughes et al., 2011). In sum, there is good evidence of the induction of ROS production by Wolbachia in novel hosts, whereas findings on the effects of novel infections on antioxidant production are so far inconclusive.

\section{Wolbachia and the Oxidative Environment in Native Hosts}

In addition to the results by Brennan et al. (2008) in an Ae. albopictus cell line, there is also evidence in support of our hypothesis that comes from whole insects. Using different methods, Molloy and Sinkins (2015) re-examined the production of ROS and antioxidants in Ae. albopictus, both in mosquito and cell lines. Interestingly, they did not find any significant difference in infected vs. uninfected lines, a finding that differs from that by Brennan et al. (2008). Nevertheless, it can similarly be interpreted as an outcome of host-symbiont coevolution, i.e., as attenuation of the immune response to Wolbachia in its natural host (immune tolerance). Thus, although it is unclear why both studies come to different results at the molecular level, the conclusion that can be drawn from them is the same: coevolution between $A e$. albopictus and its Wolbachia has led to restored redox homeostasis, either through immune interference (suggested by the results from Brennan et al., 2008) or immune tolerance (suggested by Molloy and Sinkins, 2015).

In D. simulans naturally infected with Wolbachia, total ROS levels are significantly higher in infected males than in males cured of infection. Moreover, DUOX is located in close proximity to the Wolbachia-containing vesicles (Haukedal, 2013). This suggests that the host recognizes Wolbachia as foreign and prompts an immune response involving DUOX-dependent ROS production. On the other hand, total SOD levels (including two host SODs and bacterial Fe-SOD) are also significantly higher in infected flies than in uninfected flies (Brennan et al., 2012). These findings suggest that Wolbachia infection in the natural host $D$. simulans induces not only a host immune response, but also antioxidant production.

As already mentioned, the ability of Wolbachia to interfere with the host oxidative environment might be of particular importance if the level of oxidative stress is elevated by external factors. Toxicity of the heavy metal lead is mainly attributed to its ability to generate ROS and to impair the antioxidant defense (Flora et al., 2012). When D. melanogaster is challenged by a lead-contaminated diet, flies cured of infection exhibit a strongly increased malondialdehyde content, which is a marker for oxidative stress. In addition, high-lead diet significantly decreases SOD activity in cured flies, but not in infected flies (Wang et al., 2012).

Another example of the putative role of Wolbachia in maintaining redox homeostasis under stressful conditions involves the oxidative challenge imposed by blood-feeding. Ingestion of a blood meal is associated with the release of large amounts of the iron-containing cofactor heme in the gut. When not bound to proteins, heme has potential prooxidant and cytotoxic effects in that it converts weakly ROS into highly reactive ones (Jeney et al., 2002). Hematophagous 
insects have evolved different mechanisms to be protected from these cytotoxic effects, including the binding, aggregation, and degradation of heme, and expression of antioxidant enzymes (Oliveira et al., 1999; Graça-Souza et al., 2006; Paiva-Silva et al., 2006). Maintenance of redox homeostasis in the midgut after a blood meal is crucial, not least because of the pivotal role of ROS in gut immunity. In the mosquito Ae. aegypti, a blood meal leads, perhaps counterintuitively at first, to a dramatic decrease in ROS levels in the midgut (Oliveira et al., 2011). This decrease is due to a heme-mediated activation of protein kinase $\mathrm{C}$ (PKC) which leads to lowered ROS generation in midgut epithelial cells. The authors interpret this as an adaptation to compensate for the pro-oxidant blood meal and to avoid hememediated oxidative stress, thus maintaining redox homeostasis. However, lowered ROS levels in the gut are probably associated with decreased resistance to infection and increased mortality (Oliveira et al., 2011). Interestingly, overall ROS levels do not change significantly after a blood meal in Ae. polynesiensis which, unlike Ae. aegypti, is naturally infected with Wolbachia (Andrews et al., 2012). In a coevolutionary process, the host might have abolished the PKC-mediated decrease in ROS levels in the gut because of Wolbachia-induced antioxidant production (immune interference). Therefore, it is reasonable to assume that Wolbachia help in maintaining redox homeostasis in this evolved symbiosis (Gill et al., 2014). Moreover, when Ae. polynesiensis is fed sucrose only, there is no significant difference between ROS levels of infected and cured mosquitoes, and these ROS levels are lower than that of artificially infected mosquitoes (Andrews et al., 2012). A possible explanation for this finding is that, due to coevolution between Ae. polynesiensis and its symbiont, the mosquito has reduced ROS production to mitigate oxidative stress (immune tolerance). Evolution of immune tolerance might therefore also be at play in the Wolbachia-Ae. polynesiensis symbiosis (Moné et al., 2014).

In the spider mite T. urticae, Wolbachia infection is associated with the enrichment of gene sets related to oxidoreductase activity (Zhang et al., 2015). Oxidoreductases are known to produce ROS (Raha and Robinson, 2000; Esterházy et al., 2008), but also to control redox homeostasis (Messens et al., 2013). Moreover, Wolbachia encodes an oxidoreductase ( $\alpha$-DsbA1) which, due to its low redox potential, might have antioxidant properties (Kurz et al., 2009). Therefore, it is conceivable that Wolbachia directly or indirectly regulate redox homeostasis and thus maintain their association with $T$. urticae.

We do not want to conceal that there also are some findings from natural Wolbachia-host associations that are more difficult to reconcile with the above hypothesis, or at least more difficult to interpret. In the pill bug Armadillidium vulgare, some antioxidants (thioredoxin, ferritin) are upregulated in the ovaries of infected individuals (as compared to uninfected ones), while others (Prx, glutathione peroxidase) are downregulated (Chevalier et al., 2012). Perhaps, upregulation of some antioxidants is just a compensation for the downregulation of others, or vice versa. It is unclear, however, what is induced by the host and what by the bacteria. In the parasitoid wasp Asobara tabida, the expression of several antioxidant genes (oxidoreductase, glutathione peroxidase, ferritin) is downregulated in infected ovaries, compared to ovaries from cured wasps (Kremer et al., 2012). At first, this seems to contradict our hypothesis. However, the Wolbachia-Asobara tabida association is a special case because here, the host is strictly dependent on its symbiont (Zug and Hammerstein, 2015). Females cured of infection fail to produce oocytes, due to extensive apoptosis in egg chambers (Pannebakker et al., 2007). The authors suggest a co-evolutionary scenario where the wasp responds to infection with apoptosis, which is then suppressed by Wolbachia. Asobara tabida in turn compensates for suppression by further increasing the apoptotic signal because it is essential for proper egg development (Pannebakker et al., 2007). There is good empirical support for this scenario. First, Wolbachia are probably able to directly or indirectly suppress apoptosis. Suppression of apoptosis might be due to Wolbachia interfering with host iron metabolism and oxidative stress control (Kremer et al., 2010; Gill et al., 2014; Zug and Hammerstein, 2015). Moreover, it is known that ROS can act as initiators and mediators of apoptosis (Simon et al., 2000; Dixon and Stockwell, 2014). Therefore, downregulation of antioxidant genes could be a host measure to further increase the apoptotic signal. In sum, the dependence of Asobara tabida on Wolbachia might well be a consequence of the evolution of tolerance following the disruption of redox homeostasis (Moné et al., 2014; Zug and Hammerstein, 2015).

Lastly, we point to the fact that all cases that are compatible with the hypothesis involve CI-inducing Wolbachia (Ae. albopictus, Ae. polynesiensis, D. melanogaster, D. simulans, T. urticae). In contrast, Armadillidium vulgare is naturally infected with feminizing Wolbachia, and the strain that Asobara tabida depends on for oogenesis does not exhibit any reproductive phenotype (although the possibility that it induces CI remains untested). Therefore, one could think of a mechanistic connection between the host oxidative environment and the CI phenotype. Indeed, Brennan et al. (2012) showed that total SOD levels are significantly higher in testes of $D$. simulans males infected with CI-Wolbachia than in testes of cured males. Taking this as evidence of higher oxidative stress in infected testes, the authors presumed that disruption of redox homeostasis caused DNA damage in spermatocytes of infected males. Strikingly, DNA damage is significantly higher in infected compared to uninfected spermatocytes and might be a contributing factor to the sperm modification characteristic of CI (Brennan et al., 2012). For example, DNA damage in spermatocytes could, after fertilization, lead to DNA replication defects in the male pronucleus as observed in CI crosses in D. simulans (Landmann et al., 2009).

\section{Wolbachia and Anti-Pathogenic Effects}

The possibility of Wolbachia-induced host protection has recently spurred intense research efforts. Taking a slightly critical stance, we have proposed to distinguish protection from mere antipathogenic effects (Zug and Hammerstein, 2015). Following our definition, Wolbachia are said to induce an antipathogenic effect whenever infection increases host resistance and/or tolerance to pathogens. However, an antipathogenic effect should only be classified as protection if it is associated with a fitness benefit to the host. Since so far only a few studies have found evidence for Wolbachia-mediated protection in the field 
(Hedges et al., 2008; Teixeira et al., 2008; Osborne et al., 2009; Zélé et al., 2012), we here focus on antipathogenic effects.

The molecular mechanisms underlying Wolbachia-mediated antipathogenic effects are still unclear (Rainey et al., 2014; Johnson, 2015; Zug and Hammerstein, 2015). Antiviral effects seem to be more frequent than antibacterial effects. Moreover, the strength of the antipathogenic effect is positively correlated to Wolbachia density. But how is the insect immune system involved? Antipathogenic effects are frequently observed when Wolbachia are transfected into hosts that are either naturally uninfected or infected with a different strain. As outlined above, in such cases, infection induces the upregulation of host immune genes, in particular genes involved in the Toll and Imd pathway, leading to the generation of AMPs. Such immune upregulation of Toll/Imd pathway genes is assumed to underlie antipathogenic effects in novel hosts, especially antiviral effects in mosquitoes (Xi et al., 2008a, Kambris et al., 2009, 2010; Moreira et al., 2009; Bian et al., 2010; Pan et al., 2012). However, other studies have shown that, both in native and novel hosts, genes involved in the Toll or Imd pathway are not required for Wolbachiamediated antipathogenic effects (Wong et al., 2011; Rancès et al., 2012, 2013; Chrostek et al., 2014; Ferreira et al., 2014; Martinez et al., 2014). Therefore, upregulation of immune genes involved in the Toll/Imd pathways cannot be the universal explanation for Wolbachia-induced antipathogenic effects, let alone for host protection in the field (Zug and Hammerstein, 2015).

The possible role of ROS in Wolbachia-induced antipathogenic effects has been less intensively studied than that of AMPs. The mosquito Ae. aegypti is naturally not infected with Wolbachia, but transfection of the $w$ AlbB strain into Ae. aegypti inhibits replication of Dengue virus (Bian et al., 2010). It could be shown that transfection induces NOX- and DUOX-dependent ROS generation. Increased ROS levels activate the Toll pathway, which then mediates the production of antioxidants and AMPs such as defensin and cecropin. These AMPs are involved in inhibiting the proliferation of Dengue virus in Wolbachiatransfected mosquitoes (Pan et al., 2012). In transfected Ae. albopictus mosquitoes, by contrast, ROS-mediated immune activation is probably not involved in the antiviral effect of Wolbachia (Molloy and Sinkins, 2015). A recent study analyzed the relationship between ROS levels and antiviral effects in naturally infected Drosophila strains (Wong et al., 2015). The study included Wolbachia strains that were known to either have an antipathogenic effect ("protective" strains) or not ("nonprotective" strains). In flies that harbor a protective strain, ROS levels are significantly higher than in flies cured of the protective strain. By contrast, presence of the non-protective strain has no significant effect on ROS levels relative to cured flies. These findings suggest that ROS levels are increased in Drosophila naturally infected with protective Wolbachia strains. Moreover, elevated ROS levels confer a survival advantage against mortality induced by Drosophila C virus (DCV; Wong et al., 2015). The anti-DCV effect is probably not mediated by the Toll pathway because Wolbachia-induced antiviral effects were shown to be independent of this pathway in Drosophila for both Dengue virus and DCV (Rancès et al., 2013; Ferreira et al., 2014). Interestingly, the ROS-mediated survival advantage is not associated with reduced virus accumulation, pointing to increased tolerance rather than resistance (Wong et al., 2015). Tolerance mechanisms have been shown to be at play in other coevolved Wolbachia-host systems where the symbionts induce antipathogenic effects (Teixeira et al., 2008; Osborne et al., 2009; Zélé et al., 2014). In sum, the possibility that a Wolbachia-induced ROS-based immune response is involved in antipathogenic effects constitutes a promising topic for future research.

\section{Wolbachia, ROS, Life-History Trade-Offs, and Mitohormesis}

Organisms cannot maximize all fitness-relevant traits at once. Rather, they face the challenge to optimally allocate limited resources among those traits. Hence, the evolution of fitness-related traits is constrained by the existence of trade-offs between them. These trade-offs play a fundamental role in life-history theory (Stearns, 1989). Along these lines, immune defense can be viewed as a life-history trait as well, and trade-offs between immunity and other fitness-related traits ("costs of immunity") have been gaining increasing attention among evolutionary ecologists (Sheldon and Verhulst, 1996; Zuk and Stoehr, 2002; Schmid-Hempel, 2003; Schulenburg et al., 2009; McKean and Lazzaro, 2011).

Much effort has been made to elucidate the physiological mechanisms underlying life-history trade-offs. Given their antagonistic and pleiotropic effects, ROS have recently been proposed as central players in the occurrence of such trade-offs (Dowling and Simmons, 2009; Monaghan et al., 2009; Metcalfe and Alonso-Alvarez, 2010; Isaksson et al., 2011; but see Speakman and Garratt, 2014). In particular, because of their pivotal role in innate immunity on the one hand and in oxidative stress on the other hand, ROS may be a key factor underlying the trade-off between immunity and other life-history traits such as fecundity and longevity (Moné et al., 2014).

Building upon these ideas and on the intimate connections between Wolbachia and the host oxidative environment, one may speculate that Wolbachia are involved in the occurrence of the trade-off between immunity and other life-history traits, and that this involvement is, at least in part, mediated by ROS. There is some evidence for this hypothesis. Pigeault et al. (2014) studied the effect of transfected Wolbachia strains on immunity and reproduction in the woodlouse Porcellio dilatatus. They found a clear trade-off between both life-history traits: the $w$ Con strain increases investment in immune parameters but reduces reproductive investment (whereas the $w$ Dil strain has the converse effect). However, the tested immune parameters (such as hemocyte density or phagocytosis activity) do not allow to draw a conclusion on whether ROS are involved in the trade-off. In $D$. simulans, there is a similar tradeoff between Wolbachia-induced antiviral protection and egg hatch rates, female fecundity, and male fertility (Martinez et al., 2015). Another example of Wolbachia-associated costs of immunity involves the trade-off between immunity and longevity. 
Wolbachia strains that induce strong antiviral effects in $D$. melanogaster (so-called wMelCS-like strains) often shorten the host lifespan (Chrostek et al., 2013). Strikingly, the wMelCS strain was recently shown to increase ROS concentration twofold relative to a Wolbachia-free control (Wong et al., 2015). Therefore, it is possible that elevated ROS levels are responsible not only for the antiviral effect, but also for the shortened lifespan.

The impact of ROS and oxidative stress on longevity and aging has been debated for more than half a century. The seminal "free radical theory of aging" states that the production of mitochondrial ROS is the major cause of aging (Harman, 1956, 1972; Balaban et al., 2005). However, findings are accumulating that seem to be incompatible with this theory (Lapointe and Hekimi, 2010; Speakman and Selman, 2011; Stuart et al., 2014; but see Kirkwood and Kowald, 2012). In particular, recent evidence suggests that moderately increased formation of ROS in the mitochondria causes higher stress resistance and eventually extends life span, a process that has been termed mitochondrial hormesis (mitohormesis; Ristow and Schmeisser, 2014; Yun and Finkel, 2014). In general, hormesis is defined as any adaptive response exhibiting a biphasic dose response (Calabrese and Baldwin, 2002). Usually, such biphasic dose responses are characterized by a beneficial effect at low doses and a harmful effect at higher doses. In a narrower, and recently more frequently used, sense, hormesis describes the phenomenon that a mild, sublethal stress causes an adaptive response that protects against larger subsequent stresses. The latter meaning of the term has been named "stress-response hormesis" (Gems and Partridge, 2008). Mitohormesis represents a form of stressresponse hormesis: Mild mitochondrial stress increases ROS formation which induces stress response mechanisms (such as antioxidant production), ultimately causing a long-term reduction of oxidative stress. Mitohormesis thus involves both an increase in mitochondrial ROS and a subsequent antioxidant response, and the notion of a mitohormetic pathway is tightly associated with the role of ROS as important signaling molecules (Hamanaka and Chandel, 2010; Finkel, 2011). Several recent studies have shown this mitohormetic pathway to be at work in promoting survival and longevity (Kharade et al., 2005; Chávez et al., 2007; Schulz et al., 2007; Zarse et al., 2012; Mouchiroud et al., 2013; De Haes et al., 2014). Given that Wolbachia are known to promote longevity in several hosts (Zug and Hammerstein, 2015), it is tempting to speculate that they do so by triggering the mitohormetic pathway. More generally, the mitohormetic pathway is strongly reminiscent of the hypothesized "immune interference" phenotype of Wolbachia in native hosts (in which the symbionts not only induce a ROS-based immune response, but also the expression of antioxidant genes; Figure 1F). Taken together, some fitness-enhancing effects of native Wolbachia (e.g., promoting longevity, maintaining redox homeostasis) might be attributable to mitohormesis.

With regard to the impact of ROS on fitness-related traits, the trade-off approach and the mitohormesis approach might appear to come to quite different conclusions. For example, ROS are assumed to shorten lifespan under the former approach and to extend lifespan under the latter. More generally, the trade-off approach states that Wolbachia (via ROS) have a positive effect on some fitness parameters and a negative effect on others, whereas the mitohormesis approach emphasizes the positive fitness effect of Wolbachia-induced mitochondrial ROS formation. However, hormesis itself is assumed to trade off with at least some fitness-related traits because a positive hormetic effect on overall fitness would be at odds with life-history theory (Forbes, 2000). Accordingly, a recent study finds that pathogen challenge in Drosophila enhances not only survival and fecundity, but also susceptibility to infection, suggesting a trade-off between hormesis and immunity (McClure et al., 2014). Therefore, both approaches involve some form of trade-off and thus are not mutually exclusive.

\section{CONCLUSION}

Reactive oxygen species represent a double-edged sword: They are known to cause oxidative stress and damage cellular macromolecules. However, given their cytotoxic nature, ROS also are efficient microbicidal effectors which play a crucial role in the insect immune system. Due to this antagonistic pleiotropy, ROS probably underlie evolutionary trade-offs between immunity and other life-history traits such as fecundity and longevity. Wolbachia are widespread intracellular bacteria famous for their ability to modulate exactly these fitness-related host traits in intriguing ways. At the same time, they must be able to cope with the host immune system in order to invade and persist in their insect hosts. Therefore, the host oxidative environment represents a promising area to elucidate the mechanisms of Wolbachia-host interactions.

In newly infected hosts, Wolbachia usually trigger an immune response which is aimed at eliminating the infection. In coevolved associations, by contrast, either the host has curbed the immune response when it pays to do so, or the symbionts have evolved ways to resist the host immune response. They do so by adopting a variety of strategies, including immune evasion by stealth, suppression, and interference. We propose that in co-evolved symbioses, Wolbachia frequently make use of the latter strategy in that they not only induce a ROS-based immune response but also an antioxidant response. Thereby the bacteria are involved in maintaining redox homeostasis. Interference with the host oxidative environment might also underlie other mutualistic phenotypes of Wolbachia such as enhancing host defense or promoting longevity, possibly via mitohormetic effects. On the other hand, Wolbachia-induced ROS formation might be involved in parasitic phenotypes such as cytoplasmic incompatibility. Taken together, Wolbachia's impact on the host oxidative environment probably contributed to their tremendous success and opens up exciting avenues for future research.

\section{ACKNOWLEDGMENTS}

This article was supported by the Deutsche Forschungsgemeinschaft (SFB 618, C1). We thank the two reviewers for their helpful comments and suggestions. 


\section{REFERENCES}

Aguirre, J., and Lambeth, J. D. (2010). Nox enzymes from fungus to fly to fish and what they tell us about Nox function in mammals. Free Radic. Biol. Med. 49, 1342-1353. doi: 10.1016/j.freeradbiomed.2010.07.027

Andrews, E. S., Crain, P. R., Fu, Y., Howe, D. K., and Dobson, S. L. (2012). Reactive oxygen species production and Brugia pahangi survivorship in Aedes polynesiensis with artificial Wolbachia infection types. PLoS Pathog. 8:e1003075. doi: 10.1371/journal.ppat.1003075

Bae, Y. S., Choi, M. K., and Lee, W.-J. (2010). Dual oxidase in mucosal immunity and host-microbe homeostasis. Trends Immunol. 31, 278-287. doi: 10.1016/j.it.2010.05.003

Balaban, R. S., Nemoto, S., and Finkel, T. (2005). Mitochondria, oxidants, and aging. Cell 120, 483-495. doi: 10.1016/j.cell.2005.02.001

Baldridge, G. D., Kurtti, T. J., and Munderloh, U. G. (2005). Susceptibility of rickettsia monacensis and Rickettsia peacockii to cecropin a, ceratotoxin a, and lysozyme. Curr. Microbiol. 51, 233-238. doi: 10.1007/s00284-005$4532-7$

Ballard, J. W. O. (2005). Drosophila simulans as a novel model for studying mitochondrial metabolism and aging. Exp. Gerontol. 40, 763-773. doi: 10.1016/j.exger.2005.07.014

Ballard, J. W. O., and Melvin, R. G. (2007). Tetracycline treatment influences mitochondrial metabolism and mtDNA density two generations after treatment in Drosophila. Insect. Mol. Biol. 16, 799-802. doi: 10.1111/j.1365-2583.2007.00760.x

Barnham, K. J., Masters, C. L., and Bush, A. I. (2004). Neurodegenerative diseases and oxidative stress. Nat. Rev. Drug Discov. 3, 205-214. doi: 10.1038/nrd1330

Bedard, K., Lardy, B., and Krause, K.-H. (2007). NOX family NADPH oxidases: not just in mammals. Biochimie 89, 1107-1112. doi: 10.1016/j.biochi.2007.01.012

Bergin, D., Reeves, E. P., Renwick, J., Wientjes, F. B., and Kavanagh, K. (2005). Superoxide production in Galleria mellonella hemocytes: identification of proteins homologous to the NADPH oxidase complex of human neutrophils. Infect. Immun. 73, 4161-4170. doi: 10.1128/IAI.73.7.4161-4170.2005

Bian, G., Joshi, D., Dong, Y., Lu, P., Zhou, G., Pan, X., et al. (2013). Wolbachia invades Anopheles stephensi populations and induces refractoriness to Plasmodium infection. Science 340, 748-751. doi: 10.1126/science.1236192

Bian, G., Xu, Y., Lu, P., Xie, Y., and Xi, Z. (2010). The endosymbiotic bacterium Wolbachia induces resistance to dengue virus in Aedes aegypti. PLoS Pathog. 6:e1000833. doi: 10.1371/journal.ppat.1000833

Bonnay, F., Cohen-Berros, E., Hoffmann, M., Kim, S. Y., Boulianne, G. L., Hoffmann, J. A., et al. (2013). big bang gene modulates gut immune tolerance in Drosophila. Proc. Natl Acad. Sci. U.S.A. 110, 2957-2962. doi: 10.1073/pnas. 1221910110

Bosco-Drayon, V., Poidevin, M., Boneca, I. G., Narbonne-Reveau, K., Royet, J., and Charroux, B. (2012). Peptidoglycan sensing by the receptor PGRP-LE in the Drosophila gut induces immune responses to infectious bacteria and tolerance to microbiota. Cell Host Microbe 12, 153-165. doi: 10.1016/j.chom.2012.06.002

Bourtzis, K., Pettigrew, M. M., and O'Neill, S. L. (2000). Wolbachia neither induces nor suppresses transcripts encoding antimicrobial peptides. Insect Mol. Biol. 9 , 635-639. doi: 10.1046/j.1365-2583.2000.00224.x

Brennan, C. A., and Anderson, K. V. (2004). Drosophila: the genetics of innate immune recognition and response. Annu. Rev. Immunol. 22, 457-483. doi: 10.1146/annurev.immunol.22.012703.104626

Brennan, L. J., Haukedal, J. A., Earle, J. C., Keddie, B., and Harris, H. L. (2012). Disruption of redox homeostasis leads to oxidative DNA damage in spermatocytes of Wolbachia-infected Drosophila simulans. Insect Mol. Biol. 21, 510-520. doi: 10.1111/j.1365-2583.2012.01155.x

Brennan, L. J., Keddie, B. A., Braig, H. R., and Harris, H. L. (2008). The endosymbiont Wolbachia pipientis induces the expression of host antioxidant proteins in an Aedes albopictus cell line. PLoS ONE 3:e2083. doi: 10.1371/journal.pone.0002083

Brown, D. I., and Griendling, K. K. (2009). Nox proteins in signal transduction. Free Radic. Biol. Med. 47, 1239-1253. doi: 10.1016/j.freeradbiomed.2009.07.023

Buchon, N., Broderick, N. A., and Lemaitre, B. (2013). Gut homeostasis in a microbial world: insights from Drosophila melanogaster. Nat. Rev. Microbiol. 11, 615-626. doi: 10.1038/nrmicro3074

Buchon, N., Silverman, N., and Cherry, S. (2014). Immunity in Drosophila melanogaster-from microbial recognition to whole-organism physiology. Nat. Rev. Immunol. 14, 796-810. doi: 10.1038/nri3763
Calabrese, E. J., and Baldwin, L. A. (2002). Defining hormesis. Hum. Exp. Toxicol. 21, 91-97. doi: 10.1191/0960327102ht217oa

Carrondo, M. A. (2003). Ferritins, iron uptake and storage from the bacterioferritin viewpoint. EMBO J. 22, 1959-1968. doi: 10.1093/emboj/cdg215

Carter, V., and Hurd, H. (2010). Choosing anti-Plasmodium molecules for genetically modifying mosquitoes: focus on peptides. Trends Parasitol. 26, 582-590. doi: 10.1016/j.pt.2010.07.005

Cassat, J. E., and Skaar, E. P. (2013). Iron in infection and immunity. Cell Host Microbe 13, 509-519. doi: 10.1016/j.chom.2013.04.010

Chakrabarti, S., Poidevin, M., and Lemaitre, B. (2014). The Drosophila MAPK p38c regulates oxidative stress and lipid homeostasis in the intestine. PLoS Genet. 10:e1004659. doi: 10.1371/journal.pgen.1004659

Chávez, V., Mohri-Shiomi, A., Maadani, A., Vega, L. A., and Garsin, D. A. (2007). Oxidative stress enzymes are required for DAF-16-mediated immunity due to generation of reactive oxygen species by Caenorhabditis elegans. Genetics 176, 1567-1577. doi: 10.1534/genetics.107.072587

Cheng, Q., Ruel, T. D., Zhou, W., Moloo, S. K., Majiwa, P., O'Neill, S. L., et al. (2000). Tissue distribution and prevalence of Wolbachia infections in tsetse flies, Glossina spp. Med. Vet. Entomol. 14, 44-50. doi: 10.1046/j.13652915.2000.00202.x

Chevalier, F., Herbinière-Gaboreau, J., Charif, D., Mitta, G., Gavory, F., Wincker, P., et al. (2012). Feminizing Wolbachia: a transcriptomics approach with insights on the immune response genes in Armadillidium vulgare. BMC Microbiol. 12:S1. doi: 10.1186/1471-2180-12-S1-S1

Choy, A., and Roy, C. R. (2013). Autophagy and bacterial infection: an evolving arms race. Trends Microbiol. 21, 451-456. doi: 10.1016/j.tim.2013.06.009

Chrostek, E., Marialva, M. S. P., Esteves, S. S., Weinert, L. A., Martinez, J., Jiggins, F. M., et al. (2013). Wolbachia variants induce differential protection to viruses in Drosophila melanogaster: a phenotypic and phylogenomic analysis. PLoS Genet. 9:e1003896. doi: 10.1371/journal.pgen.1003896

Chrostek, E., Marialva, M. S. P., Yamada, R., O'Neill, S. L., and Teixeira, L. (2014). High anti-viral protection without immune upregulation after interspecies Wolbachia transfer. PLoS ONE 9:e99025. doi: 10.1371/journal.pone. 0099025

Cirimotich, C. M., Dong, Y., Clayton, A. M., Sandiford, S. L., Souza-Neto, J. A., Mulenga, M., et al. (2011). Natural microbe-mediated refractoriness to Plasmodium infection in Anopheles gambiae. Science 332, 855-858. doi: 10.1126/science. 1201618

Dantoft, W., Davis, M. M., Lindvall, J. M., Tang, X., Uvell, H., Junell, A., et al. (2013). The Oct1 homolog Nubbin is a repressor of NF-kB-dependent immune gene expression that increases the tolerance to gut microbiota. BMC Biol. 11:99. doi: 10.1186/1741-7007-11-99

D’Autréaux, B., and Toledano, M. B. (2007). ROS as signalling molecules: mechanisms that generate specificity in ROS homeostasis. Nat. Rev. Mol. Cell Biol. 8, 813-824. doi: 10.1038/nrm2256

De Haes, W., Frooninckx, L., Van Assche, R., Smolders, A., Depuydt, G., Billen, J., et al. (2014). Metformin promotes lifespan through mitohormesis via the peroxiredoxin PRDX-2. Proc. Natl Acad. Sci. U.S.A. 111, E2501-E2509. doi: 10.1073/pnas.1321776111

DeJong, R. J., Miller, L. M., Molina-Cruz, A., Gupta, L., Kumar, S., and BarillasMury, C. (2007). Reactive oxygen species detoxification by catalase is a major determinant of fecundity in the mosquito Anopheles gambiae. Proc. Natl Acad. Sci. U.S.A. 103, 2121-2126. doi: 10.1073/pnas.0608407104

Deretic, V. (2010). Autophagy in infection. Curr. Opin. Cell Biol. 22, 252-262. doi: 10.1016/j.ceb.2009.12.009

Deretic, V., Saitoh, T., and Akira, S. (2013). Autophagy in infection, inflammation and immunity. Nat. Rev. Immunol. 13, 722-737. doi: 10.1038/nri3532

Diaz-Albiter, H., Sant'Anna, M. R. V., Genta, F. A., and Dillon, R. J. (2012). Reactive oxygen species-mediated immunity against Leishmania mexicana and Serratia marcescens in the phlebotomine sand fly Lutzomyia longipalpis. J. Biol. Chem. 287, 23995-24003. doi: 10.1074/jbc.M112.376095

Dixon, S. J., and Stockwell, B. R. (2014). The role of iron and reactive oxygen species in cell death. Nature Chem. Biol. 10, 9-17. doi: 10.1038/nchembio.1416

Dobson, S. L., Bourtzis, K., Braig, H. R., Jones, B. F., Zhou, W., Rousset, F., et al. (1999). Wolbachia infections are distributed throughout insect somatic and germ line tissues. Insect Biochem. Mol. Biol. 29, 153-160.

Dowling, D. K., and Simmons, L. W. (2009). Reactive oxygen species as universal constraints in life-history evolution. Proc. R. Soc. B 276, 1737-1745. doi: $10.1098 /$ rspb.2008.1791 
Driver, C., Georgiou, A., and Georgiou, G. (2004). The contribution by mitochondrially induced oxidative damage to aging in Drosophila melanogaster. Biogerontology 5, 185-192. doi: 10.1023/B:BGEN.0000031156. 75376.e3

Dunning Hotopp, J. C., Lin, M., Madupu, R., Crabtree, J., Angiuoli, S. V., Eisen, J., et al. (2006). Comparative genomics of emerging human ehrlichiosis agents. PLoS Genet. 2:e21. doi: 10.1371/journal.pgen.0020021

Engel, P., and Moran, N. (2013). The gut microbiota of insects-diversity in structure and function. FEMS Microbiol. Rev. 37, 699-735. doi: 10.1111/15746976.12025

Erkosar, B., Storelli, G., Defaye, A., and Leulier, F. (2013). Host-intestinal microbiota mutualism: "learning on the fly." Cell Host Microbe 13, 8-14. doi: 10.1016/j.chom.2012.12.004

Esterházy, D., King, M. S., Yakovlev, G., and Hirst, J. (2008). Production of reactive oxygen species by complex I (NADH: ubiquinone oxidoreductase) from Escherichia coli and comparison to the enzyme from mitochondria. Biochemistry 47, 3964-3971. doi: 10.1021/bi702243b

Fauvarque, M.-O., and Williams, M. J. (2011). Drosophila cellular immunity: a story of migration and adhesion. J. Cell Sci. 124, 1373-1382. doi: 10.1242/jcs.064592

Ferrandon, D., Imler, J.-L., Hetru, C., and Hoffmann, J. A. (2007). The Drosophila systemic immune response: sensing and signalling during bacterial and fungal infections. Nat. Rev. Immunol. 7, 862-874. doi: 10.1038/nri2194

Ferreira, Á. G., Naylor, H., Esteves, S. S., Pais, I. S., Martins, N. E., and Teixeira, L. (2014). The Toll-Dorsal pathway is required for resistance to viral oral infection in Drosophila. PLoS Pathog. 10:e1004507. doi: 10.1371/journal.ppat.1004507

Finkel, T. (2011). Signal transduction by reactive oxygen species. J. Cell Biol. 194, 7-15. doi: $10.1083 /$ jcb. 201102095

Flora, G., Gupta, D., and Tiwari, A. (2012). Toxicity of lead: a review with recent updates. Interdiscip. Toxicol. 5, 47-58. doi: 10.2478/v10102-012-0009-2

Forbes, V. E. (2000). Is hormesis an evolutionary expectation? Funct. Ecol. 14, 12-24. doi: 10.1046/j.1365-2435.2000.00392.x

Frost, C. L., Pollock, S. W., Smith, J. E., and Hughes, W. O. H. (2014). Wolbachia in the flesh: symbiont intensities in germ-line and somatic tissues challenge the conventional view of Wolbachia transmission routes. PLoS ONE 9:e95122. doi: 10.1371/journal.pone.0095122

García-Gil de Muñoz, F., Lanz-Mendoza, H., and Hernández-Hernández, F. C. (2007). Free radical generation during the activation of hemolymph prepared from the homopteran Dactylopius coccus. Arch. Insect Biochem. Physiol. 65, 20-28. doi: 10.1002/arch.20174

Gems, D., and Partridge, L. (2008). Stress-response hormesis and aging: "that which does not kill us makes us stronger." Cell Metab. 7, 200-203. doi: 10.1016/j.cmet.2008.01.001

Gerschman, R., Gilbert, D. L., Nye, S. W., Dwyer, P., and Fenn, W. O. (1954). Oxygen poisoning and X-irradiation: a mechanism in common. Science 119, 623-626.

Gill, A. C., Darby, A. C., and Makepeace, B. L. (2014). Iron necessity: the secret of Wolbachia's success? PLoS Negl. Trop. Dis. 8:e3224. doi: 10.1371/journal.pntd.0003224

Gonçalves, R. L. S., Oliveira, J. H. M., Oliveira, G. A., Andersen, J. F., Oliveira, M. F., Oliveira, P. L., et al. (2012). Mitochondrial reactive oxygen species modulate mosquito susceptibility to Plasmodium infection. PLoS ONE 7:e41083. doi: 10.1371/journal.pone.0041083

Graça-Souza, A. V., Maya-Monteiro, C., Paiva-Silva, G. O., Braz, G. R. C., Paes, M. C., Sorgine, M. H. F., et al. (2006). Adaptations against heme toxicity in blood-feeding arthropods. Insect Biochem. Mol. Biol. 36, 322-335. doi: 10.1016/j.ibmb.2006.01.009

Griendling, K. K., and FitzGerald, G. A. (2003). Oxidative stress and cardiovascular injury. Part I: basic mechanisms and in vivo monitoring of ROS. Circulation 108, 1912-1916. doi: 10.1161/01.CIR.0000093660.86242.BB

Ha, E.-M., Lee, K.-A., Park, S. H., Kim, S.-H., Nam, H.-J., Lee, H.-Y., et al. (2009a). Regulation of DUOX by the G $\alpha$ q-phospholipase $C \beta-\mathrm{Ca}^{2+}$ pathway in Drosophila gut immunity. Dev. Cell 16, 386-397. doi: 10.1016/j.devcel.2008.12.015

Ha, E.-M., Lee, K.-A., Seo, Y. Y., Kim, S.-H., Lim, J.-H., Oh, B.-H., et al. (2009b). Coordination of multiple dual oxidase-regulatory pathways in responses to commensal and infectious microbes in Drosophila gut. Nat. Immunol. 10, 949-957. doi: 10.1038/ni.1765

Ha, E.-M., Oh, C.-T., Bae, Y. S., and Lee, W.-J. (2005a). A direct role for dual oxidase in Drosophila gut immunity. Science 310, 847-850. doi: 10.1126/science.1117311
Ha, E.-M., Oh, C.-T., Ryu, J.-H., Bae, Y.-S., Kang, S.-W., Jang, I.-H., et al. (2005b). An antioxidant system required for host protection against gut infection in Drosophila. Dev. Cell 8, 125-132. doi: 10.1016/j.devcel.2004.11.007

Hamanaka, R. B., and Chandel, N. S. (2010). Mitochondrial reactive oxygen species regulate cellular signaling and dictate biological outcomes. Trends Biochem. Sci. 35, 505-513. doi: 10.1016/j.tibs.2010.04.002

Harman, D. (1956). Aging: a theory based on free radical and radiation chemistry. J. Gerontol. 11, 298-300.

Harman, D. (1972). The biologic clock: the mitochondria? J. Am. Geriatr. Soc. 20, $145-147$.

Haukedal, J. A. (2013). Regulation of Wolbachia Density Within Drosophila Simulans. Ph.D. thesis, University of Alberta, Edmonton, Alberta.

Hedges, L. M., Brownlie, J. C., O’Neill, S. L., and Johnson, K. N. (2008). Wolbachia and virus protection in insects. Science 322, 702. doi: 10.1126/science.1162418

Hetru, C., and Hoffmann, J. A. (2009). NF-кB in the immune response of Drosophila. Cold Spring Harb. Perspect. Biol. 1:a000232. doi: 10.1101/cshperspect.a000232

Hilgenboecker, K., Hammerstein, P., Schlattmann, P., Telschow, A., and Werren, J. H. (2008). How many species are infected with Wolbachia?-a statistical analysis of current data. FEMS Microbiol. Lett. 281, 215-220. doi: 10.1111/j.15746968.2008.01110.x

Ho, Y.-S., Magnenat, J.-L., Gargano, M., and Cao, J. (1998). The nature of antioxidant defense mechanisms: a lesson from transgenic studies. Environ. Health Perspect. $106,1219-1228$.

Holmström, K. M., and Finkel, T. (2014). Cellular mechanisms and physiological consequences of redox-dependent signalling. Nat. Rev. Mol. Cell Biol. 15, 411-421. doi: 10.1038/nrm3801

Hornett, E. A., Charlat, S., Duplouy, A. M. R., Davies, N., Roderick, G. K., Wedell, N., et al. (2006). Evolution of male-killer suppression in a natural population. PLoS Biol. 4:e283. doi: 10.1371/journal.pbio.0040283

Huang, J., Canadien, V., Lam, G. Y., Steinberg, B. E., Dinauer, M. C., Magalhaes, M. A. O., et al. (2009). Activation of antibacterial autophagy by NADPH oxidases. Proc. Natl Acad. Sci. U.S.A. 106, 6226-6231. doi: 10.1073/pnas.08110 45106

Hughes, G. L., Ren, X., Ramirez, J. L., Sakamoto, J. M., Bailey, J. A., Jedlicka, A. E., et al. (2011). Wolbachia infections in Anopheles gambiae cells: transcriptomic characterization of a novel host-smbiont interaction. PLoS Pathog. 7:e1001296. doi: 10.1371/journal.ppat.1001296

Huigens, M. E. (2003). On the Evolution of Wolbachia-induced Parthenogenesis in Trichogramma Wasps. Ph.D. thesis, Wageningen University, Wageningen, Netherlands.

Hurst, G. D. D., Anbutsu, H., Kutsukake, M., and Fukatsu, T. (2003). Hidden from the host: Spiroplasma bacteria infecting Drosophila do not cause an immune response, but are suppressed by ectopic immune activation. Insect Mol. Biol. 12, 93-97. doi: 10.1046/j.1365-2583.2003.00380.x

Hurst, G. D. D., and Jiggins, F. M. (2005). Problems with mitochondrial DNA as a marker in population, phylogeographic and phylogenetic studies: the effects of inherited symbionts. Proc. R. Soc. B 272, 1525-1534. doi: 10.1098/rspb.2005.3056

Hutchence, K. J., Fischer, B., Paterson, S., and Hurst, G. D. D. (2011). How do insects react to novel inherited symbionts? A microarray analysis of Drosophila melanogaster response to the presence of natural and introduced Spiroplasma. Mol. Ecol. 20, 950-958. doi: 10.1111/j.1365-294X.2010. 04974.x

Ijichi, N., Kondo, N., Matsumoto, R., Shimada, M., Ishikawa, H., and Fukatsu, T. (2002). Internal spatiotemporal population dynamics of infection with three Wolbachia strains in the adzuki bean beetle, Callosobruchus chinensis (Coleoptera: Bruchidae). Appl. Environ. Microbiol. 68, 4074-4080. doi: 10.1128/AEM.68.8.4074-4080.2002

Isaksson, C., Sheldon, B. C., and Uller, T. (2011). The challenges of integrating oxidative stress into life-history biology. Bioscience 61, 194-202. doi: 10.1525/bio.2011.61.3.5

Ishii, K., Hamamoto, H., Kamimura, M., and Sekimizu, K. (2008). Activation of the silkworm cytokine by bacterial and fungal cell wall components via a reactive oxygen species-triggered mechanism. J. Biol. Chem. 283, 2185-2191. doi: $10.1074 /$ jbc.M705480200

Jeney, V., Balla, J., Yachie, A., Varga, Z., Vercellotti, G. M., Eaton, J. W., et al. (2002). Pro-oxidant and cytotoxic effects of circulating heme. Blood 100, 879-887. doi: 10.1182/blood.V100.3.879 
Jiravanichpaisal, P., Lee, B. L., and Söderhäll, K. (2006). Cell-mediated immunity in arthropods: hematopoiesis, coagulation, melanization and opsonization. Immunobiology 211, 213-236. doi: 10.1016/j.imbio.2005.10.015

Johnson, K. N. (2015). Bacteria and antiviral immunity in insects. Curr. Opin. Insect Sci. 8, 97-103. doi: 10.1016/j.cois.2015.01.008

Kambris, Z., Blagborough, A. M., Pinto, S. B., Blagrove, M. S. C., Godfray, H. C. J., Sinden, R. E., et al. (2010). Wolbachia stimulates immune gene expression and inhibits Plasmodium development in Anopheles gambiae. PLoS Pathog. 6:e1001143. doi: 10.1371/journal.ppat.1001143

Kambris, Z., Cook, P. E., Phuc, H. K., and Sinkins, S. P. (2009). Immune activation by life-shortening Wolbachia and reduced filarial competence in mosquitoes. Science 326, 134-136. doi: 10.1126/science.1177531

Kaneko, T., Yano, T., Aggarwal, K., Lim, J.-H., Ueda, K., Oshima, Y., et al. (2006). PGRP-LC and PGRP-LE have essential yet distinct functions in the Drosophila immune response to monomeric DAP-type peptidoglycan. Nat. Immunol. 7, 715-723. doi: $10.1038 /$ ni1356

Kharade, S. V., Mittal, N., Das, S. P., Sinha, P., and Roy, N. (2005). Mrg19 depletion increases $S$. cerevisiae lifespan by augmenting ROS defence. FEBS Lett. 579, 6809-6813. doi: 10.1016/j.febslet.2005.11.017

Khush, R. S., Leulier, F., and Lemaitre, B. (2001). Drosophila immunity: two paths to NF-кB. Trends Immunol. 22, 260-264. doi: 10.1016/S1471-4906(01)01887-7

Kim, S.-H., and Lee, W.-J. (2014). Role of DUOX in gut inflammation: lessons from Drosophila model of gut-microbiota interactions. Front. Cell Infect. Microbiol. 3:116. doi: 10.3389/fcimb.2013.00116

Kirkwood, T. B. L., and Kowald, A. (2012). The free-radical theory of ageing-older, wiser and still alive. Bioessays 34, 692-700. doi: 10.1002/bies.201200014

Koehncke, A., Telschow, A., Werren, J. H., and Hammerstein, P. (2009). Life and death of an influential passenger: Wolbachia and the evolution of CI-modifiers by their hosts. PLoS ONE 4:e4425. doi: 10.1371/journal.pone.0004425

Kremer, N., Charif, D., Henri, H., Gavory, F., Wincker, P., Mavingui, P., et al. (2012). Influence of Wolbachia on host gene expression in an obligatory symbiosis. BMC Microbiol. 12:S7. doi: 10.1186/1471-2180-12-S1-S7

Kremer, N., Dedeine, F., Charif, D., Finet, C., Allemand, R., and Vavre, F. (2010). Do variable compensatory mechanisms explain the polymorphism of the dependence phenotype in the Asobara tabida-Wolbachia association? Evolution 64, 2969-2979. doi: 10.1111/j.1558-5646.2010.01034.x

Kremer, N., Voronin, D., Charif, D., Mavingui, P., Mollereau, B., and Vavre, F. (2009). Wolbachia interferes with ferritin expression and iron metabolism in insects. PLoS Pathog. 5:e1000630. doi: 10.1371/journal.ppat. 1000630

Kumar, S., Christophides, G. K., Cantera, R., Charles, B., Han, Y. S., Meister, S., et al. (2003). The role of reactive oxygen species on Plasmodium melanotic encapsulation in Anopheles gambiae. Proc. Natl Acad. Sci. U.S.A. 100, 14139-14144. doi: 10.1073/pnas.2036262100

Kuraishi, T., Hori, A., and Kurata, S. (2013). Host-microbe interactions in the gut of Drosophila melanogaster. Front. Physiol. 4:375. doi: 10.3389/fphys.2013.00375

Kurata, S. (2010). Extracellular and intracellular pathogen recognition by Drosophila PGRP-LE and PGRP-LC. Int. Immunol. 22, 143-148. doi: 10.1093/intimm/dxp128

Kurz, M., Iturbe-Ormaetxe, I., Jarrott, R., Shouldice, S. R., Wouters, M. A., Frei, P., et al. (2009). Structural and functional characterization of the oxidoreductase a-DsbA1 from Wolbachia pipientis. Antioxid. Redox Signal. 11, 1485-1500. doi: 10.1089/ars.2008.2420

Lambeth, J. D. (2004). Nox enzymes and the biology of reactive oxygen. Nat. Rev. Immunol. 4, 181-189. doi: 10.1038/nri1312

Lambeth, J. D., and Neish, A. S. (2014). Nox enzymes and new thinking on reactive oxygen: a double-edged sword revisited. Annu. Rev. Pathol. Mech. Dis. 9, 119-145. doi: 10.1146/annurev-pathol-012513-104651

Lamiable, O., and Imler, J.-L. (2014). Induced antiviral innate immunity in Drosophila. Curr. Opin. Microbiol. 20, 62-68. doi: 10.1016/j.mib.2014.05.006

Landmann, F., Orsi, G. A., Loppin, B., and Sullivan, W. J. (2009). Wolbachiamediated cytoplasmic incompatibility is associated with impaired histone deposition in the male pronucleus. PLoS Pathog. 5:e1000343. doi: 10.1371/journal.ppat.1000343

Lapointe, J., and Hekimi, S. (2010). When a theory of aging ages badly. Cell. Mol. Life Sci. 67, 1-8. doi: 10.1007/s00018-009-0138-8

Le Clec'h, W., Braquart-Varnier, C., Raimond, M., Ferdy, J.-B., Bouchon, D., and Sicard, M. (2012). High virulence of Wolbachia after host switching: when autophagy hurts. PLoS Pathog. 8:e1002844. doi: 10.1371/journal.ppat.1002844
Lee, K.-A, Kim, B., Bhin, J., Kim, D. H., You, H., Kim, E.-K., et al. (2015). Bacterial uracil modulates Drosophila DUOX-dependent gut immunity via Hedgehog-induced signaling endosomes. Cell Host Microbe 17, 191-204. doi: 10.1016/j.chom.2014.12.012

Lee, K.-A., Kim, S.-H., Kim, E.-K., Ha, E.-M., You, H., Kim, B., et al. (2013). Bacterial-derived uracil as a modulator of mucosal immunity and gut-microbe homeostasis in Drosophila. Cell 153, 797-811. doi: 10.1016/j.cell.2013. 04.009

Lee, W.-J., and Brey, P. T. (2013). How microbiomes influence metazoan development: insights from history and Drosophila modeling of gut-microbe interactions. Annu. Rev. Cell Dev. Biol. 29, 571-592. doi: 10.1146/annurevcellbio-101512-122333

Lemaitre, B., and Hoffmann, J. (2007). The host defense of Drosophila melanogaster. Annu. Rev. Immunol. 25, 697-743. doi: 10.1146/annurev.immunol.25.022106.141615

Leulier, F., Parquet, C., Pili-Floury, S., Ryu, J. H., Caroff, M., Lee, W.-J., et al. (2003). The Drosophila immune system detects bacteria through specific peptidoglycan recognition. Nat. Immunol. 4, 478-484. doi: 10.1038/ni922

Lhocine, N., Ribeiro, P. S., Buchon, N., Wepf, A., Wilson, R., Tenev, T., et al. (2008). PIMS modulates immune tolerance by negatively regulating Drosophila innate immune signaling. Cell Host Microbe 4, 147-158. doi: 10.1016/j.chom.2008.07.004

Liu, H., Bao, W., Lin, M., Niu, H., and Rikihisa, Y. (2012a). Ehrlichia type IV secretion effector ECH0825 is translocated to mitochondria and curbs ROS and apoptosis by upregulating host MnSOD. Cell. Microbiol. 14, 1037-1050. doi: 10.1111/j.1462-5822.2012.01775.x

Liu, L., Dai, J., Zhao, Y. O., Narasimhan, S., Yang, Y., Zhang, L., et al. (2012b). Ixodes scapularis JAK-STAT pathway regulates tick antimicrobial peptides, thereby controlling the agent of human granulocytic anaplasmosis. J. Infect. Dis. 206, 1233-1241. doi: 10.1093/infdis/jis484

Login, F. H., Balmand, S., Vallier, A., Vincent-Monégat, C., Vigneron, A., Weiss-Gayet, M., et al. (2011). Antimicrobial peptides keep insect endosymbionts under control. Science 334, 362-365. doi: 10.1126/science.12 09728

Louis, C., and Nigro, L. (1989). Ultrastructural evidence of Wolbachia Rickettsiales in Drosophila simulans and their relationships with unidirectional cross-incompatibility. J. Invertebr. Pathol. 54, 39-44. doi: 10.1016/0022-2011(89)90137-7

Lundgren, J. G., and Jurat-Fuentes, J. L. (2012). "Physiology and ecology of host defense against microbial invaders," in Insect Pathology, Second Edition, eds F. E. Vega and H. K. Kaya (San Diego, CA: Academic Press), 461-480.

Maritim, A. C., Sanders, R. A., and Watkins III, J. B. (2003). Diabetes, oxidative stress, and antioxidants: a review. J. Biochem. Mol. Toxicol. 17, 24-38. doi: $10.1002 /$ jbt. 10058

Martinez, J., Longdon, B., Bauer, S., Chan, Y.-S., Miller, W. J., Bourtzis, K., et al. (2014). Symbionts commonly provide broad spectrum resistance to viruses in insects: a comparative analysis of Wolbachia strains. PLoS Pathog. 10:e1004369. doi: 10.1371/journal.ppat.1004369

Martinez, J., Ok, S., Smith, S., Snoeck, K., Day, J. P., and Jiggins, F. M. (2015). Should symbionts be nice or selfish? Antiviral effects of Wolbachia are costly but reproductive parasitism is not. PLoS Pathog. 11:e1005021. doi: 10.1371/journal.ppat.1005021

Masri, L., and Cremer, S. (2014). Individual and social immunisation in insects. Trends Immunol. 35, 471-482. doi: 10.1016/j.it.2014.08.005

Mateos, M., Castrezana, S. J., Nankivell, B. J., Estes, A. M., Markow, T. A., and Moran, N. A. (2006). Heritable endosymbionts of Drosophila. Genetics 174, 363-376. doi: 10.1534/genetics.106.058818

McClure, C. D., Zhong, W., Hunt, V. L., Chapman, F. M., Hill, F. V., and Priest, N. K. (2014). Hormesis results in trade-offs with immunity. Evolution 68, 2225-2233. doi: 10.1111 /evo. 12453

McCord, J. M., and Fridovich, I. (1969). Superoxide dismutase. An enzymic function for erythrocuprein (hemocuprein). J. Biol. Chem. 244, 6049-6055.

McCord, J. M., Keele, B. B. Jr, and Fridovich, I. (1971). An enzyme-based theory of obligate anaerobiosis: the physiological function of superoxide dismutase. Proc. Natl Acad. Sci. U.S.A. 68, 1024-1027. doi: 10.1073/pnas.68. 5.1024

McKean, K. A., and Lazzaro, B. (2011). "The costs of immunity and the evolution of immunological defense mechanisms," in Mechanisms of Life History Evolution, eds T. Flatt and A. Heyland (Oxford: Oxford University Press), 299-310. 
Merkling, S. H., and van Rij, R. P. (2013). Beyond RNAi: antiviral defense strategies in Drosophila and mosquito. J. Insect Physiol. 59, 159-170. doi: 10.1016/j.jinsphys.2012.07.004

Messens, J., Rouhier, N., and Collet, J.-F. (2013). “Redox Homeostasis,” in Oxidative Stress and Redox Regulation, eds U. Jakob and D. Reichmann (Dordrecht: Springer), 59-84.

Metcalfe, N. B., and Alonso-Alvarez, C. (2010). Oxidative stress as a lifehistory constraint: the role of reactive oxygen species in shaping phenotypes from conception to death. Funct. Ecol. 24, 984-996. doi: 10.1111/j.13652435.2010.01750.x

Mikonranta, L., Mappes, J., Kaukoniitty, M., and Freitak, D. (2014). Insect immunity: oral exposure to a bacterial pathogen elicits free radical response and protects from a recurring infection. Front. Zool. 11:23. doi: 10.1186/1742-999411-23

Molina-Cruz, A., DeJong, R. J., Charles, B., Gupta, L., Kumar, S., JaramilloGutierrez, G., et al. (2008). Reactive oxygen species modulate Anopheles gambiae immunity against bacteria and Plasmodium. J. Biol. Chem. 283, 3217-3223. doi: 10.1074/jbc.M705873200

Molloy, J. C., and Sinkins, S. P. (2015). Wolbachia do not induce reactive oxygen species-dependent immune pathway activation in Aedes albopictus. Viruses 7, 4624-4639. doi: 10.3390/v7082836

Monaghan, P., Metcalfe, N. B., and Torres, R. (2009). Oxidative stress as a mediator of life history trade-offs: mechanisms, measurements and interpretation. Ecol. Lett. 12, 75-92. doi: 10.1111/j.1461-0248.2008.01258.x

Moné, Y., Monnin, D., and Kremer, N. (2014). The oxidative environment: a mediator of interspecies communication that drives symbiosis evolution. Proc. R. Soc. B 281, 20133112. doi: 10.1098/rspb.2013.3112

Moreira, L. A., Iturbe-Ormaetxe, I., Jeffery, J. A., Lu, G., Pyke, A. T., Hedges, L. M., et al. (2009). A Wolbachia symbiont in Aedes aegypti limits infection with Dengue, Chikungunya, and Plasmodium. Cell 139, 1268-1278. doi: 10.1016/j.cell.2009.11.042

Mouchiroud, L., Houtkopper, R. H., Moullan, N., Katsyuba, E., Ryu, D., Cantó, C., et al. (2013). The NAD+/sirtuin pathway modulates longevity through activation of mitochondrial UPR and FOXO signaling. Cell 154, 430-441. doi: 10.1016/j.cell.2013.06.016

Nairz, M., Haschka, D., Demetz, E., and Weiss, G. (2014). Iron at the interface of immunity and infection. Front. Pharmacol. 5:152. doi: 10.3389/fphar.2014.00152

Nakamoto, M., Moy, R. H., Xu, J., Bambina, S., Yasunaga, A., Shelly, S. S., et al. (2012). Virus recognition by Toll-7 activates antiviral autophagy in Drosophila. Immunity 36, 658-667. doi: 10.1016/j.immuni.2012.03.003

Nappi, A. J., and Vass, E. (1998). Hydrogen peroxide production in immune-reactive Drosophila melanogaster. J. Parasitol. 84, 1150-1157.

Nappi, A. J., and Vass, E. (2002). Interactions of iron with reactive intermediates of oxygen and nitrogen. Dev. Neurosci. 24, 134-142. doi: 10.1159/000 065697

Nappi, A. J., Vass, E., Frey, F., and Carton, Y. (1995). Superoxide anion generation in Drosophila during melanotic encapsulation of parasites. Eur. J. Cell Biol. 68, 450-456.

Nathan, C., and Cunningham-Bussel, A. (2013). Beyond oxidative stress: an immunologist's guide to reactive oxygen species. Nat. Rev. Immunol. 13, 349-361. doi: 10.1038/nri3423

Neyen, C., Poidevin, M., Roussel, A., and Lemaitre, B. (2012). Tissue- and ligandspecific sensing of Gram-negative infection in Drosophila by PGRP-LC isoforms and PGRP-LE. J. Biol. Chem. 189, 1886-1897. doi: 10.4049/jimmunol.1201022

Oliveira, J. H. M., Gonçalves, R. L. S., Lara, F. A., Dias, F. A., Gandara, A. C. P., Menna-Barreto, R. F. S., et al. (2011). Blood meal-derived heme decreases ROS levels in the midgut of Aedes aegypti and allows proliferation of intestinal microbiota. PLoS Pathog. 7:e1001320. doi: 10.1371/journal.ppat. 1001320

Oliveira, M. F., Silva, J. R., Dansa-Petretski, M., de Souza, W., Lins, U., Braga, C. M. S., et al. (1999). Haem detoxification by an insect. Nature 400, 517-518. doi: $10.1038 / 22910$

Osborne, S. E., Leong, Y. S., O’Neill, S. L., and Johnson, K. N. (2009). Variation in antiviral protection mediated by different Wolbachia strains in Drosophila simulans. PLoS Pathog. 5:e1000656. doi: 10.1371/journal.ppat.1000656

Paiva-Silva, G. O., Cruz-Oliveira, C., Nakayasu, E. S., Maya-Monteiro, C. M., Dunkov, B. C., Masuda, H., et al. (2006). A heme-degradation pathway in a blood-sucking insect. Proc. Natl Acad. Sci. U.S.A. 103, 8030-8035. doi: $10.1073 /$ pnas.0602224103
Pan, X., Zhou, G., Wu, J., Bian, G., Lu, P., Raikhel, A. S., et al. (2012). Wolbachia induces reactive oxygen species (ROS)-dependent activation of the Toll pathway to control dengue virus in the mosquito Aedes aegypti. Proc. Natl Acad. Sci. U.S.A. 109, E23-E31. doi: 10.1073/pnas.1116932108

Pannebakker, B. A., Loppin, B., Elemans, C. P. H., Humblot, L., and Vavre, F. (2007). Parasitic inhibition of cell death facilitates symbiosis. Proc. Natl Acad. Sci. U.S.A. 104, 213-215. doi: 10.1073/pnas.0607845104

Paredes, J. C., Welchman, D. P., Poidevin, M., and Lemaitre, B. (2011). Negative regulation by amidase PGRPs shapes the Drosophila antibacterial response and protects the fly from innocuous infection. Immunity 35, 770-779. doi: 10.1016/j.immuni.2011.09.018

Parkes, T. L., Kirby, K., Phillips, J. P., and Hilliker, A. J. (1998). Transgenic analysis of the cSOD-null phenotypic syndrome in Drosophila. Genome 41, 642-651.

Parsons, L. M., Lin, F., and Orban, J. (2006). Peptidoglycan recognition by Pal, an outer membrane lipoprotein. Biochemistry 45, 2122-2128. doi: 10.1021/bi052227i

Péan, C. B., and Dionne, M. S. (2014). Intracellular infections in Drosophila melanogaster: host defense and mechanisms of pathogenesis. Dev. Comp. Immunol. 42, 57-66. doi: 10.1016/j.dci.2013.04.013

Pereira, L. S., Oliviera, P. L., Barja-Fidalgo, C., and Daffre, S. (2001). Production of reactive oxygen species by hemocytes from the cattle tick Boophilus microplus. Exp. Parasitol. 99, 66-72. doi: 10.1006/expr.2001.4657

Pichon, S., Bouchon, D., Cordaux, R., Chen, L., Garrett, R. A., and Grève, P. (2009). Conservation of the type IV secretion system throughout Wolbachia evolution. Biochem. Biophys. Res. Commun. 385, 557-562. doi: 10.1016/j.bbrc.2009.05.118

Pigeault, R., Braquart-Varnier, C., Marcadé, I., Mappa, G., Mottin, E., and Sicard, M. (2014). Modulation of host immunity and reproduction by horizontally acquired Wolbachia. J. Insect Physiol. 70, 125-133. doi: 10.1016/j.jinsphys.2014.07.005

Raha, S., and Robinson, B. H. (2000). Mitochondria, oxygen free radicals, disease and ageing. Trends Biochem. Sci. 25, 502-508. doi: 10.1016/S09680004(00)01674-1

Rainey, S. M., Shah, P., Kohl, A., and Dietrich, I. (2014). Understanding the Wolbachia-mediated inhibition of arboviruses in mosquitoes: progress and challenges. J. Gen. Virol. 95, 517-530. doi: 10.1099/vir.0.057422-0

Rancès, E., Johnson, T. K., Popovici, J., Iturbe-Ormaetxe, I., Zakir, T., Warr, C. G., et al. (2013). The Toll and Imd pathways are not required for Wolbachia-mediated dengue virus interference. J. Virol. 87, 11945-11949. doi: 10.1128/JVI.01522-13

Rancès, E., Ye, Y. H., Woolfit, M., McGraw, E. A., and O’Neill, S. L. (2012). The relative importance of innate immune priming in Wolbachia-mediated dengue interference. PLoS Pathog. 8:e1002548. doi: 10.1371/journal.ppat.1002548

Ray, P. D., Huang, B.-W., and Tsuji, Y. (2012). Reactive oxygen species (ROS) homeostasis and redox regulation in cellular signaling. Cell. Signal. 24, 981-990. doi: 10.1016/j.cellsig.2012.01.008

Reczek, C. R., and Chandel, N. S. (2015). ROS-dependent signal transduction. Curr Opin. Cell Biol. 33, 8-13. doi: 10.1016/j.ceb.2014.09.010

Rigaud, T., and Juchault, P. (1992). Genetic control of the vertical transmission of a cytoplasmic sex factor in Armadillidium vulgare Latr. (Crustacea, Oniscidea). Heredity 68, 47-52.

Ristow, M., and Schmeisser, K. (2014). Mitohormesis: promoting health and lifespan by increased levels of reactive oxygen species (ROS). Dose Resp. 12, 288-341. doi: 10.2203/dose-response.13-035.Ristow

Rolff, J., and Reynolds, S. E., eds. (2009). Insect Infection and Immunity. Oxford: Oxford University Press.

Roth, O., Sadd, B. M., Schmid-Hempel, P., and Kurtz, J. (2009). Strain-specific priming of resistance in the red flour beetle, Tribolium castaneum. Proc. R. Soc. B 276, 145-151. doi: 10.1098/rspb.2008.1157

Royer-Pokora, B., Kunkel, L. M., Monaco, A. P., Goff, S. C., Newburger, P. E., Baehner, R. L., et al. (1986). Cloning of the gene for an inherited human disorder-chronic granulomatous disease-on the basis of its chromosomal location. Nature 322, 32-38. doi: 10.1038/322032a0

Royet, J., and Dziarski, R. (2007). Peptidoglycan recognition proteins: pleiotropic sensors and effectors of antimicrobial defences. Nat. Rev. Microbiol. 5, 264-277. doi: $10.1038 /$ nrmicrol620

Royet, J., Gupta, D., and Dziarski, R. (2011). Peptidoglycan recognition proteins: modulators of the microbiome and inflammation. Nat. Rev. Immunol. 11, 837-851. doi: 10.1038/nri3089

Ryu, J.-H., Ha, E.-M., and Lee, W.-J. (2010). Innate immunity and gut-microbe mutualism in Drosophila. Dev. Comp. Immunol. 34, 369-376. doi: 10.1016/j.dci.2009.11.010 
Ryu, J.-H., Ha, E.-M., Oh, C.-T., Seol, J.-H., Brey, P. T., Jin, I., et al. (2006). An essential complementary role of NF- $\kappa B$ pathway to microbicidal oxidants in Drosophila gut immunity. EMBO J. 25, 3693-3701. doi: 10.1038/sj.emboj.7601233

Ryu, J.-H., Kim, S.-H., Lee, H.-Y., Bai, J. Y., Nam, Y.-D., Bae, J.-W., et al. (2008). Innate immune homeostasis by the homeobox gene Caudal and commensal-gut mutualism in Drosophila. Science 319, 777-782. doi: 10.1126/science.1149357

Sabin, L. R., Hanna, S. L., and Cherry, S. (2010). Innate antiviral immunity in Drosophila. Curr. Opin. Immunol. 22, 4-9. doi: 10.1016/j.coi.2010.01.007

Sansonetti, P. J., and Medzhitov, R. (2009). Learning tolerance while fighting ignorance. Cell 138, 416-420. doi: 10.1016/j.cell.2009.07.024

Scherz-Shouval, R., and Elazar, Z. (2011). Regulation of autophagy by ROS: physiology and pathology. Trends Biochem. Sci. 36, 30-38. doi: 10.1016/j.tibs.2010.07.007

Schieber, M., and Chandel, N. S. (2014). ROS function in redox signaling and oxidative stress. Curr. Biol. 24, R453-R462. doi: 10.1016/j.cub.2014.03.034

Schmid-Hempel, P. (2003). Variation in immune defence as a question of evolutionary ecology. Proc. R. Soc. B 270, 357-366. doi: 10.1098/rspb.2002.2265

Schneider, D. S., and Ayres, J. S. (2008). Two ways to survive infection: what resistance and tolerance can teach us about treating infectious diseases. Nat. Rev. Immunol. 8, 889-895. doi: 10.1038/nri2432

Schulenburg, H., Kurtz, J., Moret, Y., and Siva-Jothy, M. T. (2009). Introduction. Ecological immunology. Phil. Trans. R. Soc. B 364, 3-14. doi: 10.1098/rstb.2008.0249

Schulz, T. J., Zarse, K., Voigt, A., Urban, N., Birringer, M., and Ristow, M. (2007). Glucose restriction extends Caenorhabditis elegans life span by inducing mitochondrial respiration and increasing oxidative stress. Cell Metab. 6, 280-293. doi: 10.1016/j.cmet.2007.08.011

Sena, L. A., and Chandel, N. S. (2012). Physiological roles of mitochondrial reactive oxygen species. Mol. Cell 48, 158-167. doi: 10.1016/j.molcel.2012.09.025

Sheldon, B. C., and Verhulst, S. (1996). Ecological immunology: costly parasite defences and trade-offs in evolutionary ecology. Trends Ecol. Evol. 11, 317-321. doi: 10.1016/0169-5347(96)10039-2

Simon, H.-U., Haj-Yehia, A., and Levi-Schaffer, F. (2000). Role of reactive oxygen species (ROS) in apoptosis induction. Apoptosis 5, 415-418. doi: 10.1023/A:1009616228304

Siozios, S., Sapountzis, P., Ioannidis, P., and Bourtzis, K. (2008). Wolbachia symbiosis and insect immune response. Insect Sci. 15, 89-100. doi: 10.1111/j.1744-7917.2008.00189.x

Sommer, F., and Bäckhed, F. (2013). The gut microbiota-masters of host development and physiology. Nat. Rev. Microbiol. 11, 227-238. doi: 10.1038/nrmicro2974

Speakman, J. R., and Garratt, M. (2014). Oxidative stress as a cost of reproduction: beyond the simplistic trade-off model. Bioessays 36, 93-106. doi: 10.1002/bies.201300108

Speakman, J. R., and Selman, C. (2011). The free-radical damage theory: accumulating evidence against a simple link of oxidative stress to ageing and lifespan. Bioessays 33, 255-259. doi: 10.1002/bies.201000132

Stearns, S. C. (1989). Trade-offs in life-history evolution. Funct. Ecol. 3, 259-268.

Steinert, S., and Levashina, E. A. (2011). Intracellular immune responses of dipteran insects. Immunol. Rev. 240, 129-140. doi: 10.1111/j.1600-065X.2010. 00985.x

Storz, P. (2005). Reactive oxygen species in tumor progression. Front. Biosci. 10, 1881-1896. doi: 10.2741/1667

Stouthamer, R., Breeuwer, J. A. J., and Hurst, G. D. D. (1999). Wolbachia pipientis: microbial manipulator of arthropod reproduction. Annu. Rev. Microbiol. 53, 71-102. doi: 10.1146/annurev.micro.53.1.71

Strand, M. R. (2008). The insect cellular immune response. Insect Sci. 15, 1-14. doi: 10.1111/j.1744-7917.2008.00183.x

Stuart, J. A., Maddalena, L. A., Merilovich, M., and Robb, E. L. (2014). A midlife crisis for the mitochondrial free radical theory of aging. Longev. Healthspan 3, 4. doi: 10.1186/2046-2395-3-4

Suh, Y.-A., Arnold, R. S., Lassegue, B., Shi, J., Xu, X., Sorescu, D., et al. (1999). Cell transformation by the superoxide-generating oxidase Mox1. Nature 401, 79-82. doi: $10.1038 / 43459$

Teixeira, L., Ferreira, A., and Ashburner, M. (2008). The bacterial symbiont Wolbachia induces resistance to RNA viral infections in Drosophila melanogaster. PLoS Biol. 6:e1000002. doi: 10.1371/journal.pbio. 1000002
Tzou, P., Ohresser, S., Ferrandon, D., Capovilla, M., Reichhart, J. M., Lemaitre, B., et al. (2000). Tissue-specific inducible expression of antimicrobial peptide genes in Drosophila surface epithelia. Immunity 13, 737-748. doi: 10.1016/S10747613(00)00072-8

Valko, M., Rhodes, C. J., Moncol, J., Izakovic, M., and Mazur, M. (2006). Free radicals, metals and antioxidants in oxidative stress-induced cancer. Chem. Biol. Interact. 160, 1-40. doi: 10.1016/j.cbi.2005.12.009

Virgin, H. W., and Levine, B. (2009). Autophagy genes in immunity. Nat. Immunol. 10, 461-470. doi: 10.1038/ni.1726

Vollmer, J., Schiefer, A., Schneider, T., Jülicher, K., Johnston, K. L., Taylor, M. J., et al. (2013). Requirement of lipid II biosynthesis for cell division in cell wallless Wolbachia, endobacteria of arthropods and filarial nematodes. Int. J. Med. Microbiol. 303, 140-149. doi: 10.1016/j.ijmm.2013.01.002

Voronin, D., Cook, D. A. N., Steven, A., and Taylor, M. J. (2012). Autophagy regulates Wolbachia populations across diverse symbiotic associations. Proc. Natl Acad. Sci. U.S.A. 109, E1638-E1646. doi: 10.1073/pnas.1203519109

Voronin, D., Guimarães, A. F., Molyneux, G. R., Johnston, K. L., Ford, L., and Taylor, M. J. (2014). Wolbachia lipoproteins: abundance, localisation and serology of Wolbachia peptidoglycan associated lipoprotein and the Type IV Secretion System component, VirB6 from Brugia malayi and Aedes albopictus. Parasit. Vectors 7, 462. doi: 10.1186/s13071-014-0462-1

Wang, Y., Oberley, L. W., and Murhammer, D. W. (2001). Evidence of oxidative stress following the viral infection of two lepidopteran insect cell lines. Free Radic. Biol. Med. 31, 1448-1455. doi: 10.1016/S0891-5849(01)00728-6

Wang, L., Zhou, C., He, Z., Wang, Z.-G., Wang, J.-L., and Wang, Y.-F. (2012). Wolbachia infection decreased the resistance of Drosophila to lead. PLoS ONE 7:e32643. doi: 10.1371/journal.pone. 0032643

Weinert, L. A., Araujo-Jnr, E. V., Ahmed, M. Z., and Welch, J. J. (2015). The incidence of bacterial endosymbionts in terrestrial arthropods. Proc. R. Soc. B 282, 20150249. doi: 10.1098/rspb.2015.0249

Werren, J. H., Baldo, L., and Clark, M. E. (2008). Wolbachia: master manipulators of invertebrate biology. Nat. Rev. Microbiol. 6, 741-751. doi: 10.1038/nrmicro1969

Whitten, M. M. A., and Ratcliffe, N. A. (1999). In vitro superoxide activity in the haemolymph of the West Indian leaf cockroach, Blaberus discoidalis. J. Insect. Physiol. 45, 667-675. doi: 10.1016/S0022-1910(99)00039-6

Wong, Z. S., Brownlie, J. C., and Johnson, K. N. (2015). Oxidative stress correlates with Wolbachia-mediated antiviral protection in WolbachiaDrosophila associations. Appl. Environ. Microbiol. 81, 3001-3005. doi: 10.1128/AEM.03847-14

Wong, Z. S., Hedges, L. M., Brownlie, J. C., and Johnson, K. N. (2011). Wolbachiamediated antibacterial protection and immune gene regulation in Drosophila. PLoS ONE 6:e25430. doi: 10.1371/journal.pone.0025430

Wu, S.-C., Liao, C.-W., Pan, R.-L., and Juang, J.-L. (2012). Infectioninduced intestinal oxidative stress triggers organ-to-organ immunological communication in Drosophila. Cell Host Microbe 11, 410-417. doi: 10.1016/j.chom.2012.03.004

Xi, Z., Gavotte, L., Xie, Y., and Dobson, S. L. (2008a). Genome-wide analysis of the interaction between the endosymbiotic bacterium Wolbachia and its Drosophila host. BMC Genomics 9:1. doi: 10.1186/1471-2164-9-1

Xi, Z., Ramirez, J. L., and Dimopoulos, G. (2008b). The Aedes aegypti Toll pathway controls dengue virus infection. PLoS Pathog. 4:e1000098. doi: 10.1371/journal.ppat.1000098

Yano, T., Mita, S., Ohmori, H., Oshima, Y., Fujimoto, Y., Ueda, R., et al. (2008). Autophagic control of Listeria through intracellular innate immune recognition in Drosophila. Nat. Immunol. 9, 908-916. doi: 10.1038/ni.1634

Yi, H.-Y., Chowdhury, M., Huang, Y.-D., and Yu, X.-Q. (2014). Insect antimicrobial peptides and their applications. Appl. Microbiol. Biotechnol. 98, 5807-5822. doi: 10.1007/s00253-014-5792-6

You, H., Lee, W. J., and Lee, W.-J. (2014). Homeostasis between gut-associated microorganisms and the immune system in Drosophila. Curr. Opin. Immunol. 30, 48-53. doi: 10.1016/j.coi.2014.06.006

Yuk, J.-M., Yoshimori, T., and Jo, E.-K. (2012). Autophagy and bacterial infectious diseases. Exp. Mol. Med. 44, 99-108. doi: 10.3858/emm.2012.44.2.032

Yun, J., and Finkel, T. (2014). Mitohormesis. Cell Metab. 19, 757-766. doi: 10.1016/j.cmet.2014.01.011

Zarse, K., Schmeisser, S., Groth, M., Priebe, S., Beuster, G., Kuhlow, D., et al. (2012). Impaired insulin/IGF1 signaling extends life span by promoting mitochondrial L-proline catabolism to induce a transient ROS signal. Cell Metab. 15, 451-465. doi: 10.1016/j.cmet.2012.02.013 
Zélé, F., Nicot, A., Duron, O., and Rivero, A. (2012). Infection with Wolbachia protects mosquitoes against Plasmodium-induced mortality in a natural system. J. Evol. Biol. 25, 1243-1252. doi: 10.1111/j.1420-9101.2012. 02519.x

Zélé, F., Nicot, A., Berthomieu, A., Weill, M., Duron, O., and Rivero, A. (2014). Wolbachia increases susceptibility to Plasmodium infection in a natural system. Proc. R. Soc. B. 281, 20132837. doi: 10.1098/rspb.2013. 2837

Zhang, Y.-K., Ding, X.-L, Rong, X., and Hong, X.-Y. (2015). How do hosts react to endosymbionts? A new insight into the molecular mechanisms underlying the Wolbachia-host association. Insect Mol. Biol. 24, 1-12. doi: 10.1111/imb. 12128

Zouache, K., Voronin, D., Tran-Van, V., Mousson, L., Failloux, A.-B., and Mavingui, P. (2009). Persistent Wolbachia and cultivable bacteria infection in the reproductive and somatic tissues of the mosquito vector Aedes albopictus. PLoS ONE 4:e6388. doi: 10.1371/journal.pone. 0006388

Zug, R., and Hammerstein, P. (2012). Still a host of hosts for Wolbachia: analysis of recent data suggests that $40 \%$ of terrestrial arthropod species are infected. PLoS ONE 7:e38544. doi: 10.1371/journal.pone.0038544
Zug, R., and Hammerstein, P. (2015). Bad guys turned nice? A critical assessment of Wolbachia mutualisms in arthropod hosts. Biol. Rev. 90, 89-111. doi: 10.1111/brv. 12098

Zug, R., Koehncke, A., and Hammerstein, P. (2012). Epidemiology in evolutionary time: the case of Wolbachia horizontal transmission between arthropod host species. J. Evol. Biol. 25, 2149-2160. doi: 10.1111/j.1420-9101.2012.02601.x

Zuk, M., and Stoehr, A. M. (2002). Immune defense and host life history. Am. Nat. 160, S9-S22. doi: $10.1086 / 342131$

Conflict of Interest Statement: The authors declare that the research was conducted in the absence of any commercial or financial relationships that could be construed as a potential conflict of interest.

Copyright ( 12015 Zug and Hammerstein. This is an open-access article distributed under the terms of the Creative Commons Attribution License (CC BY). The use, distribution or reproduction in other forums is permitted, provided the original author(s) or licensor are credited and that the original publication in this journal is cited, in accordance with accepted academic practice. No use, distribution or reproduction is permitted which does not comply with these terms. 